\title{
The characteristics and structure of extra-tropical cyclones in a warmer climate
}

\author{
Victoria A. Sinclair, Mika Rantanen, Päivi Haapanala, Jouni Räisänen, and Heikki Järvinen \\ Institute for Atmospheric and Earth System Research/Physics, Faculty of Science, \\ P.O. Box 64, University of Helsinki, 00014 Helsinki, Finland
}

Correspondence: Victoria A. Sinclair (victoria.sinclair@helsinki.fi)

Received: 23 August 2019 - Discussion started: 27 August 2019

Revised: 6 November 2019 - Accepted: 22 November 2019 - Published: 3 January 2020

\begin{abstract}
Little is known about how the structure of extratropical cyclones will change in the future. In this study aquaplanet simulations are performed with a full-complexity atmospheric model. These experiments can be considered an intermediate step towards increasing knowledge of how, and why, extra-tropical cyclones respond to warming. A control simulation and a warm simulation in which the sea surface temperatures are increased uniformly by $4 \mathrm{~K}$ are run for 11 years. Extra-tropical cyclones are tracked, cyclone composites created, and the omega equation applied to assess causes of changes in vertical motion. Warming leads to a $3.3 \%$ decrease in the number of extra-tropical cyclones, with no change to the median intensity or lifetime of extra-tropical cyclones but to a broadening of the intensity distribution resulting in both more stronger and more weaker storms. Composites of the strongest extra-tropical cyclones show that total column water vapour increases everywhere relative to the cyclone centre and that precipitation increases by up to $50 \%$ with the $4 \mathrm{~K}$ warming. The spatial structure of the composite cyclone changes with warming: the $900-700 \mathrm{hPa}$ layer averaged potential vorticity, $700 \mathrm{hPa}$ ascent, and precipitation maximums associated with the warm front all move polewards and downstream, and the area of ascent expands in the downstream direction. Increases in ascent forced by diabatic heating and thermal advection are responsible for the displacement, whereas increases in ascent due to vorticity advection lead to the downstream expansion. Finally, maximum values of ascent due to vorticity advection and thermal advection weaken slightly with warming, whereas those attributed
\end{abstract}

to diabatic heating increase. Thus, cyclones in warmer climates are more diabatically driven.

\section{Introduction}

Extra-tropical cyclones (also referred to as mid-latitude cyclones) are a fundamental part of the atmospheric circulation in the mid-latitudes due to their ability to transport large amounts of heat, moisture, and momentum. Climatologically, extra-tropical cyclones are responsible for most of the precipitation in the mid-latitudes, with over $70 \%$ of precipitation in large parts of Europe and North America due to the passage of an extra-tropical cyclone (Hawcroft et al., 2012). Extra-tropical cyclones are also the primary cause of midlatitude weather variability and can lead to strong winds. For example, a severe extra-tropical cyclone Kyrill moved over large parts of northern Europe in 2007, bringing strong winds that resulted in 43 deaths and USD 6.7 billion of insured damages (Fink et al., 2009). Intense extra-tropical cyclones can also be associated with heavy rain or snow, which can result in floods and travel disruption. Thus, given the large social and economic impacts that extra-tropical cyclones can cause, there is considerable research devoted to understanding the climatology and governing dynamics of these systems.

Many studies have investigated the spatial distribution and frequency of extra-tropical cyclones in the current climate by analysing reanalysis data sets (e.g. Simmonds and Keay, 2000; Hoskins and Hodges, 2002; Wernli and Schwierz, 
2006) and consequently the location of the climatological mean storm tracks, in both hemispheres, in the current climate is well known. Climatologies of cyclone number and intensity in the current climate have also been created based on reanalysis data sets and numerous different objective cyclone tracking algorithms (Neu et al., 2013). Globally there is good agreement between methods for inter-annual variability of cyclone numbers and the shape of the cyclone intensity distribution but less agreement in terms of the total cyclone numbers, particularly in terms of weak cyclones.

The spatial structure of extra-tropical cyclones in the current climate has also been extensively examined (see Schultz et al., 2019, for an overview). The starting point was the development of the Norwegian cyclone model (Bjerknes, 1919), a conceptual framework describing the spatial and temporal evolution of an extra-tropical cyclone. As considerable variability was noted in cyclone structures, Shapiro and Keyser (1990) subsequently developed a sister conceptual model. In a different theme, Harrold (1973), Browning et al. (1973), and Carlson (1980) studied three-dimensional movement of airstreams within extra-tropical cyclones, thus developing the conveyor belt model of extra-tropical cyclones. This model incorporates warm and cold conveyor belts, which are now accepted and well-studied aspects of extra-tropical cyclones (e.g. Thorncroft et al., 1993; Wernli and Davies, 1997; Eckhardt et al., 2004; Binder et al., 2016). Recently, the structure of intense extra-tropical cyclones in reanalysis data sets has been examined in a quantitative manner by creating cyclone composites (e.g. Bengtsson et al., 2009; Catto et al., 2010; Dacre et al., 2012). Cyclone composites have also been created using satellite observations of cloud fraction and precipitation (Field and Wood, 2007; Naud et al., 2010; Govekar et al., 2014; Naud et al., 2018), which enables cyclone structure in both reanalysis and model simulations to be systematically evaluated. Thus, considerable knowledge now exists of the spatial structure, dynamics, and variability of the major precipitation-producing airstreams within extra-tropical cyclones.

A key question is then how the intensity, number, structure, and weather, for example precipitation, associated with extra-tropical cyclones will change in the future as the climate warms. To answer this question, projections from climate models can be analysed. However, how the circulation responds to warming, which includes the characteristics of extra-tropical cyclones, is notably less clear and more uncertain than the bulk, global mean thermodynamic response (Shepherd, 2014). Champion et al. (2011) investigated the impact of warming on extra-tropical cyclone properties with one global climate model by comparing historical (19802000) simulations to future (2080-2100) simulations forced by the IPCC A1B scenario. They found small, yet statistically significant, changes to the $850 \mathrm{hPa}$ maximum vorticity, with the number of extreme cyclones increasing slightly in the future and the number of average-intensity cyclones decreasing. Zappa et al. (2013b) analysed output from 19 mod- els that participated in Phase 5 of the Coupled Model Intercomparison Project (CMIP5) and compared 30-year periods of the historical (1976-2005) present-day simulations and the future climate simulations (2070-2099) forced by the Representative Concentration Pathway 4.5 (RCP4.5) and 8.5 (RCP8.5) scenarios. In the RCP4.5 scenario, Zappa et al. (2013b) found a $3.6 \%$ reduction in the total number of extra-tropical cyclones in winter, a reduction in the number of extra-tropical cyclones associated with strong $850 \mathrm{hPa}$ wind speeds, and an increase in cyclone-related precipitation. In addition, they also note that considerable variability in the response was found between different CMIP5 models. In a similar study, Chang et al. (2012) show that CMIP5 models predict a significant increase in the frequency of extreme extra-tropical cyclones during the winter in the Southern Hemisphere but a significant decrease in the most intense extra-tropical cyclones in winter in the Northern Hemisphere. A similar result was obtained by Michaelis et al. (2017), who used a mesoscale model to perform pseudo-global warming simulations over the North Atlantic where the initial and boundary condition temperatures were warmed to a degree consistent with predictions from climate models forced with RCP8.5. They find a reduction in the number of strong storms with warming and an increase in cyclone precipitation.

Models participating in CMIP5 have systematic biases in the location of the climatological storm tracks in historical simulations, particularly in the North Atlantic where the storm track tends to be either too zonal or displaced southward (Zappa et al., 2013a). In addition, the response of the storm track to warming has been found to be correlated to the characteristics of the storm track in historical simulations. Chang et al. (2012) show that in the Northern Hemisphere individual models with stronger storm tracks in historical simulations project weaker changes with warming compared to individual models with weaker historical storm tracks. Moreover, the same study shows that in the Southern Hemisphere individual models with large equatorward biases in storm track latitude predict larger poleward shifts with warming.

In order to increase confidence in climate model projections of the number and intensity of extra-tropical cyclones, there is a clear need to better understand the physical mechanisms causing changes to these weather systems. This is difficult to do based on climate model output alone as fully coupled climate models are very complex, include numerous feedbacks and non-linear interactions, and due to computational and data storage limitations offer somewhat limited model output fields with limited temporal frequency. Therefore in this study we undertake an idealized "climate change" experiment using a state-of-the-art model but configured as an aqua planet.

Idealized studies have been used extensively in the past to understand the dynamics of extra-tropical cyclones. For example, baroclinic wave simulations have been performed to understand the dynamics of extra-tropical cyclones and 
fronts in the current climate (e.g. Simmons and Hoskins, 1978; Thorncroft et al., 1993; Schemm et al., 2013; Sinclair and Keyser, 2015). More recently baroclinic life cycle experiments have also been used to assess, in a highly controlled simulation environment, how the dynamics and structure of extra-tropical cyclones may respond to climate change. Given that diabatic processes, and in particular latent heating due to condensation of water vapour, play a large role in the evolution of extra-tropical cyclones (e.g. Stoelinga, 1996), many idealized studies have focused on how the intensity and structure of extra-tropical cyclones change as temperature and moisture content are varied (e.g Boutle et al., 2011; Booth et al., 2013, 2015; Kirshbaum et al., 2018). These studies show that when moisture is increased from low levels to values typical of today's climate, extra-tropical cyclones become more intense. This is a relatively robust result across many studies and can be understood to be a consequence of an induced low-level cyclonic vorticity anomaly beneath a localized maximum in diabatic heating (Hoskins et al., 1985). However, when temperatures and moisture content are increased to values higher than in the current climate, baroclinic life cycle experiments show divergent results. For example, Rantanen et al. (2019) found that uniform warming acts to decrease both the eddy kinetic energy and the minimum surface pressure of the cyclone, whereas Kirshbaum et al. (2018) showed that for large temperature increases with constant relative humidity the eddy kinetic energy decreases whereas the minimum surface pressure increases. Furthermore, Tierney et al. (2018) documented non-monotonic behaviour of the cyclone intensity in terms of both maximum eddy kinetic energy and minimum mean surface pressure with increasing temperature.

A disadvantage of baroclinic life cycle experiments is that often only one cyclone and its response to environmental changes are considered, whereas in reality there is considerable variability in the structure, intensity, size, and lifetime of extra-tropical cyclones. Recent baroclinic life cycle studies have suggested that the response of cyclones to warming in these types of simulations may depend on how the simulation is configured (Kirshbaum et al., 2018). An alternative, yet still idealized approach, is to perform multi-year aqua-planet simulations in which thousands of extra-tropical cyclones develop and can be analysed. A benefit of this approach compared to baroclinic life cycle experiments is that experimental set-up and initial conditions have a much weaker influence on the evolution of the model state and thus on the structure and size of the simulated extra-tropical cyclones. Pfahl et al. (2015) used a simplified general circulation model in an aqua-planet configuration with a slab ocean to assess how the intensity, size, deepening rates, lifetime, and spatial structure of extra-tropical cyclones respond when the longwave optical thickness is varied in such a way that the global mean near-surface air temperature varies from 270 to $316 \mathrm{~K}$. Their main result was that changes in cyclone characteristics are relatively small except for the intensity of the strongest cy- clones, which considerably increased in strength with warming. However, this study was based on an idealized general circulation model, which contained simplified physics parameterizations; for example, the large-scale microphysical parameterization only considers the vapour-liquid phase transition.

The first aim of this study is to determine how the number, intensity, and structure of extra-tropical cyclones change in response to horizontally uniform warming. The second aim is to identify the physical mechanisms which lead to changes in vertical motion and precipitation patterns associated with extra-tropical cyclones. These aims are addressed in an idealized modelling context as it is anticipated that mechanisms will be easier to identify than in complex, fully coupled climate model simulations. In particular, a full complexity atmospheric model is used to perform two aqua-planet simulations: a control simulation and an experiment where the sea surface temperatures are uniformly warmed. Extra-tropical cyclones are then tracked and cyclone centred composites are created. The omega equation is used to determine the forcing mechanisms for vertical motion at different locations relative to the cyclone centre and at different points in the cyclone life cycle for extra-tropical cyclones in both the control and warm experiments.

The remainder of this paper is set out as follows. In Sect. 2, the full-complexity numerical model, OpenIFS, which is used in this study, is described along with the numerical experiments that are performed. In Sect. 3, the cyclone tracking scheme and the omega equation diagnostic tool, which are applied to the model output to assist with analysis, are described. The results are presented in Sects. 4 to 7 . The largescale zonal mean state and its response to warming are given briefly in Sect. 4, and the results concerning changes to bulk cyclone statistics are discussed in Sect. 5. The results concerning changes to cyclone structure as ascertained from the cyclone composites are presented in Sect. 6 and the impact of warming on the asymmetry of vertical motion in extratropical cyclones is considered in Sect. 7. The conclusions are presented and discussed in Sect. 8.

\section{OpenIFS and numerical simulations}

\subsection{Numerical model: OpenIFS}

The numerical simulations are performed with OpenIFS, which is a portable version of the Integrated Forecast System (IFS) developed and used for operational forecasting at the European Centre for Medium Range Forecasting (ECMWF). Since 2013, OpenIFS has been available under license for use by academic and research institutions. The dynamical core and physical parameterizations in OpenIFS are identical to those in the full IFS as are the land surface model and wave model. However, unlike the full IFS, OpenIFS does not have any data assimilation capacity. The 
version of OpenIFS used here (Cy40r1) was operational at ECMWF between November 2013 and May 2015. The full documentation of Cy40r1 is available online (ECMWF, 2015).

\subsection{Experiments}

Numerical simulations are performed with OpenIFS configured as an aqua planet. The surface of the Earth is therefore all ocean, and the sea surface temperatures (SSTs) are specified at the start of the simulation and held constant throughout the simulation. There is no ocean model included. However, the dynamics and physical parameterizations are exactly the same as in the full IFS and the wave model is also active in the aqua-planet simulations.

The control simulation (CNTL) is set up similarly to the experiments proposed by Neale and Hoskins (2000), and their QObs sea surface temperature distribution is used. This SST distribution is specified by a simple geometric function and is intended to resemble observed SSTs more so than the other distributions specified by Neale and Hoskins (2000). The resulting SST pattern is zonally uniform and symmetric about the Equator. The maximum SST is $27^{\circ} \mathrm{C}$ in the tropics, and poleward of $60^{\circ} \mathrm{N}$ in both hemispheres the SSTs are set to $0^{\circ} \mathrm{C}$. There is no sea ice in the simulation. The atmospheric state is initialized from a randomly selected real analysis produced at ECMWF. First the real analysis is modified by changing the land-sea mask and setting the surface geopotential to zero everywhere. The atmospheric fields are then interpolated to the new flat surface in regions where there is topography on Earth. The perturbed experiment (hereinafter referred to as SST4) is identical to CNTL except that the SSTs are uniformly warmed by $4 \mathrm{~K}$ everywhere. Both experiments have a diurnal cycle in incoming solar radiation but no annual cycle; throughout the simulations the incoming solar radiation is fixed at the equinoctial value and is thus symmetric about the Equator.

Both aqua-planet simulations are run at $\mathrm{T} 159$ resolution (approximate grid spacing of $1.125^{\circ}$ equivalent to $125 \mathrm{~km}$ ) and with 60 model levels. The model top is located at $0.1 \mathrm{hPa}$. Both simulations are run for a total of 11 years and the first year of each simulation is discarded to ensure that the model has reached a balanced state. Analysis of global precipitation from the first year of simulation (not shown) reveals that a steady state is achieved after 3-4 months. Model output, including temperature tendencies from all physical parameterization schemes, is saved every $3 \mathrm{~h}$.

\section{Analysis methods}

\subsection{Cyclone tracking and compositing}

In both numerical experiments, extra-tropical cyclones are tracked using an objective cyclone identification and tracking algorithm, TRACK (Hodges, 1994, 1995). Extra-tropical cy- clones are identified as localized maxima in the $850 \mathrm{hPa}$ relative vorticity truncated to T42 spectral resolution based on 6-hourly output from OpenIFS. All cyclones in the Northern Hemisphere are initially tracked; however to ensure that no tropical cyclones are included in the analysis, tracks which do not have at least one point north of $20^{\circ} \mathrm{N}$ are excluded. Furthermore, to ensure that only synoptic-scale, mobile systems are considered, it is required that a cyclone track last for at least $2 \mathrm{~d}$ and travel at least $1000 \mathrm{~km}$. Finally cyclones which have a maximum vorticity of less than $1 \times 10^{-5} \mathrm{~s}^{-1}$ are also excluded from the analysis. The output from TRACK consists of the longitude, latitude, and relative vorticity value of each point (every $6 \mathrm{~h}$ ) along each individual extra-tropical cyclone track from which statistics such as genesis and lysis regions may be determined.

The cyclone tracks are then used as the basis to create composites of extra-tropical cyclones following the same method as Catto et al. (2010) and Dacre et al. (2012). Rather than creating a composite of all identified extra-tropical cyclones, only the 200 strongest cyclones in terms of their maximum $850 \mathrm{hPa}$ relative vorticity are selected from the CNTL and SST4 experiments, and composites of a range of meteorological variables are created for these extreme cyclones at different offset times relative to the time of maximum intensity $(t=0 \mathrm{~h})$. Each composite is created by first determining the values of the relevant meteorological variable, at each offset time, and for each individual cyclone to be included in the composite, on a spherical grid centred on the cyclone centre. The meteorological values are thus interpolated from the native model longitude-latitude grid to this spherical grid, which has a radius of $12^{\circ}$ and is decomposed into 40 grid points in the radial direction and 360 grid points in the angular direction. To reduce smoothing errors, the cyclones are rotated so that all travel due east. To obtain the cyclone composite, the meteorological values on the radial grid are averaged at each offset time. Thus, the composite extra-tropical cyclone is the simple arithmetic mean of the 200 individual, rotated cyclones.

In addition to composites of the 200 strongest extratropical cyclones, composites of the 200 "most average" cyclones were also created for both the CNTL and SST4 experiments. These cyclones were identified as the 100 cyclones with maximum vorticity values lower than but closest to the median relative vorticity and the 100 cyclones with maximum vorticity values higher than but closest to the median relative vorticity. The results from these median composites are shown in the Supplement as although uniformly warming the SSTs led to increases in the total column water vapour and precipitation, it had little coherent impact on the spatial structure of the median extra-tropical cyclone.

\subsection{Omega equation}

The omega equation is a diagnostic equation from which the vertical motion $(\omega)$ resulting from different physical pro- 

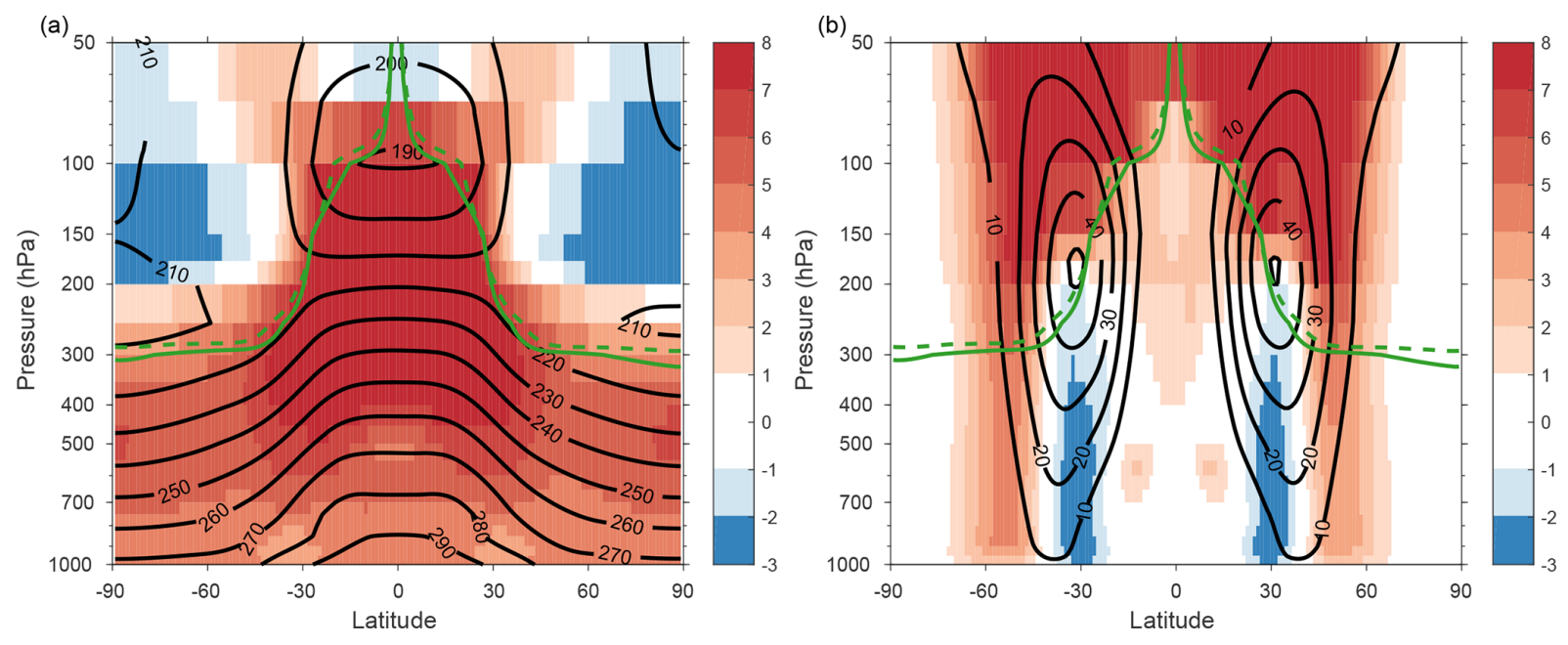

Figure 1. Zonal mean (a) temperature $(\mathrm{K})$ and (b) zonal winds $\left(\mathrm{m} \mathrm{s}^{-1}\right)$ averaged over 10 years of simulation. Black contours show the CNTL simulation and shading the difference between the SST4 and control simulations (SST4 - CNTL). The green solid line shows the dynamic tropopause (the 2 PVU surface) in CNTL and the dashed line in SST4.

cesses can be calculated. Different forms of the omega equation with differing degrees of complexity exist and range from the simplest "standard" quasi-geostrophic (QG) form with friction and diabatic heating neglected (Holton and Hakim, 2012) to the complex generalized omega equation (Räisänen, 1995; Rantanen et al., 2017). Here we solve the following version of the QG omega equation in pressure $(p)$ coordinates:

$$
\begin{aligned}
\sigma_{0}(p) \nabla^{2} \omega+f^{2} \frac{\partial^{2} \omega}{\partial p^{2}} & =f \frac{\partial}{\partial p}(\boldsymbol{v} \cdot \nabla(\zeta+f)) \\
& +\frac{R}{p} \nabla^{2}(\boldsymbol{v} \cdot \nabla T)-\frac{R}{c_{\mathrm{p}} p} \nabla^{2} Q .
\end{aligned}
$$

The static stability parameter, $\sigma_{0}$, is only a function of pressure and time and is given by

$\sigma_{0}(p)=\frac{-R T_{0}}{p} \frac{1}{\theta_{0}} \frac{\partial \theta_{0}}{\partial p}$

where $\theta_{0}\left(T_{0}\right)$ is the horizontally averaged potential temperature (temperature) profile over the global domain calculated at every time step. The left-hand side operator of Eq. (1) is identical to the standard QG omega equation. The terms on the right-hand side of Eq. (1) represent forcing for vertical motion due to differential vorticity advection, thermal advection, and diabatic heating. The right-hand side differs from the standard QG omega equation in that diabatic heating $(Q)$ is retained, the advection terms are calculated using the full horizontal winds $(v)$ rather than the geostrophic winds, and the full relative vorticity $(\zeta)$ is advected rather than the geostrophic vorticity. Friction is neglected as on an aqua planet this is expected to be small.

Overall good agreement is found between the model calculated vertical motion and the vertical motion diagnosed by
Eq. (1). Correlation coefficients between the model calculated vertical motion and the diagnosed vertical motion were calculated at each grid box and pressure level and averaged over latitude bands (not shown). In the latitude band 30$60^{\circ} \mathrm{N}$ the correlation coefficients were 0.84 at $700 \mathrm{hPa}$ and exceeded 0.9 at $500 \mathrm{hPa}$.

\section{Climatology and large-scale response to warming}

In this section the zonal mean climatology of CNTL is described along with the response to the uniform warming. Figure 1 shows that the control simulation produces a realistic distribution of temperature and of zonal winds. The dynamic tropopause varies from $300 \mathrm{hPa}$ in the polar regions to about $100 \mathrm{hPa}$ in the tropics, similar to what is observed on Earth. The zonal mean jet streams have maximum wind speeds of $45 \mathrm{~m} \mathrm{~s}^{-1}$ and are located on the tropopause at $35^{\circ} \mathrm{N} / \mathrm{S}$. As expected from the aqua-planet model set-up the two hemispheres are almost symmetrically identical.

The response to the uniform $4 \mathrm{~K}$ warming is shown by the shading in Fig. 1. The temperature increases everywhere in the troposphere with the largest warming in the tropical upper troposphere, where temperature increases by up to $7 \mathrm{~K}$. Cooling takes place in the polar stratosphere, which acts to increase the upper-level meridional temperature gradient. The tropopause height increases at most latitudes with warming. The spatial pattern of these changes in zonal mean temperature is similar to those found in more complex climate models (e.g. Fig. 12.12, Collins et al., 2013). However, the warming in the low to mid-troposphere is relatively uniform with latitude. The lack of enhanced warming in the Northern Hemisphere polar regions (polar amplification) and hence no decrease in low-level baroclinicity is the most notable differ- 
ence in the atmosphere's response to warming in these aquaplanet experiments compared to in complex climate model simulations.

At low levels, the increase in temperature in the SST4 experiment relative to CNTL is typically of the order of $4 \mathrm{~K}$, which is of similar magnitude to the enforced increase in SSTs. This temperature increase can be put into context by comparison with predictions from CMIP5 models. Under the RCP8.5 scenario, CMIP5 models predict that global mean near-surface temperatures will increase by 2.6 to $4.8 \mathrm{~K}$ by the end of the 21st century relative to the 1986-2005 mean. Hence, the aqua-planet simulations performed here have a degree of warming that could be expected to occur by the end of the 21 st century under large greenhouse gas emissions.

The response of the zonal mean zonal wind shows that the subtropical jet intensifies and moves vertically upwards. The eddy-driven jet, evident at low levels, displays a dipole structure indicative of a poleward shift. This is confirmed when the latitude of the maximum $700 \mathrm{hPa}$ zonal mean zonal wind speed is considered: this moves polewards by $3.3^{\circ}$ in the SST4 experiment compared to in CNTL. These responses of the zonal mean jet streams to uniform warming are similar to those found in more complex climate models (e.g. Collins et al., 2013), particularly in the Southern Hemisphere, demonstrating that the OpenIFS aqua planet can realistically simulate an Earth-like atmosphere.

The zonal mean precipitation in both CNTL and SST4 experiments are shown in Fig. 2a. Again strong similarities exist with real Earth observations and CMIP5 model projections (e.g. Lau et al., 2013). The largest rainfall is observed in the tropics and a secondary peak occurs in the mid-latitudes, which is associated with the mid-latitude storm track. The effect of warming the SSTs is to increase the mean precipitation at almost all latitudes. The largest absolute increase occurs in the tropics. In the Northern Hemisphere mid-latitudes the maximum precipitation rate increases from 3.9 to $4.2 \mathrm{~mm} \mathrm{~d}^{-1}$ and the location of the maximum moves polewards by $2.2^{\circ}$. This is in agreement with the poleward shift in the eddy-driven jet and strongly suggests that, on average, extra-tropical cyclones move poleward with warming. This will be confirmed in Sect. 5 .

Figure $2 \mathrm{~b}$ shows the zonal mean mean sea level pressure (MSLP). The highest zonal mean MSLP in the CNTL experiment occurs in the subtropics and moves poleward with warming. The lowest values of MSLP occur on the poleward side of the jet stream and again move poleward with warming. A notable difference between these MSLP distributions and the MSLP distribution on Earth is the absolute magnitude of the values. The mean MSLP on Earth is $1013 \mathrm{hPa}$, whereas in both the CNTL and SST4 experiments, the global mean MSLP is $985.4 \mathrm{hPa}$. This difference is solely due to the initialization method (see Sect. 2.2), and the average surface pressure of $985.4 \mathrm{hPa}$ results, as it is the average pressure at the actual surface height in the randomly selected analysis used for the initialization. Figure $2 \mathrm{c}$ shows the zonal (a)

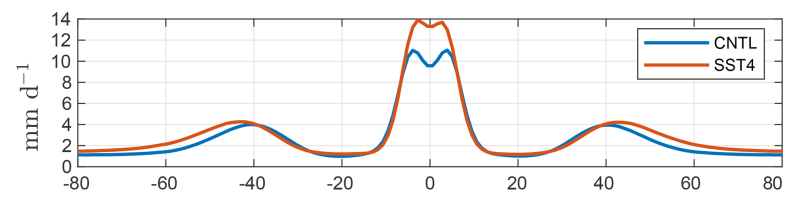

(b)

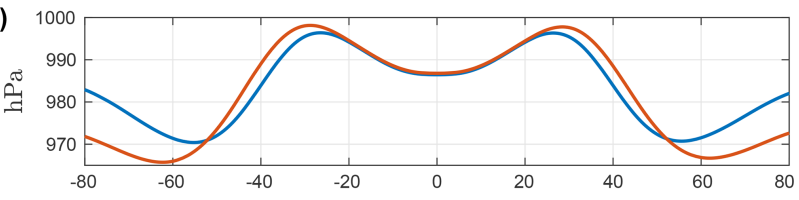

(c)

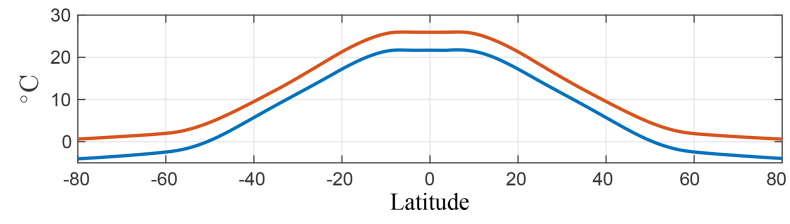

Figure 2. Zonal mean (a) total precipitation $\left(\mathrm{mm} \mathrm{d}^{-1}\right)$, (b) mean sea level pressure $(\mathrm{hPa})$, and (c) $950 \mathrm{hPa}$ temperature $\left({ }^{\circ} \mathrm{C}\right)$ averaged over 10 years of simulation. Blue line shows CNTL and red SST4.

mean $950 \mathrm{hPa}$ temperature, which indicates that the low-level temperature increase is almost constant with latitude and implies that the low-level baroclinicity does not change.

The impact of uniformly warming the SSTs on the baroclinicity can be quantified via the maximum (dry) Eady growth rate, $\sigma$, which is given by

$\sigma=0.31 \frac{|f|}{N}\left|\frac{\partial u}{\partial z}\right|$,

where $f$ is the Coriolis parameter, $N$ is the Brunt-Väisälä frequency, and $u$ is the zonal wind component. In the CNTL simulation (Fig. 3a) the Eady growth rate has maximum values of $0.75 \mathrm{day}^{-1}$ in the mid-latitude middle to upper troposphere. A secondary maximum is evident in the stratosphere; however, this most likely has little significance for the growth of extra-tropical cyclones. The response of the Eady growth rate to warming includes an increase just above the dynamical tropopause and a decrease co-located with the secondary maximum in the stratosphere. With the midtroposphere, the Eady growth rate decreases slightly with warming; for example, at $700 \mathrm{hPa}$ the maximum value decreases from $0.54 \mathrm{~d}^{-1}$ in CNTL to $0.50 \mathrm{~d}^{-1}$ in SST4. Close to the surface, at $900 \mathrm{hPa}$, the maximum value of the Eady growth rate also experiences a small decrease with warming, from 0.92 to $0.89 \mathrm{~d}^{-1}$. The most notable impact of warming on the Eady growth rate at $900 \mathrm{hPa}$ is a poleward shift of $5.4^{\circ}$ in the position of the maximum. Equatorward of $45^{\circ} \mathrm{N}$ the $900 \mathrm{hPa}$ Eady growth rate decreases with warming, whereas poleward of $45^{\circ} \mathrm{N}$ it increases.

Figure $3 \mathrm{~b}$ and $\mathrm{c}$ show the vertical shear of the zonal wind and the Brunt-Väisälä frequency respectively. There is little change in the vertical wind shear with warming in the mid-troposphere, which via thermal wind balance is consistent with the lack of any large changes to the horizontal 

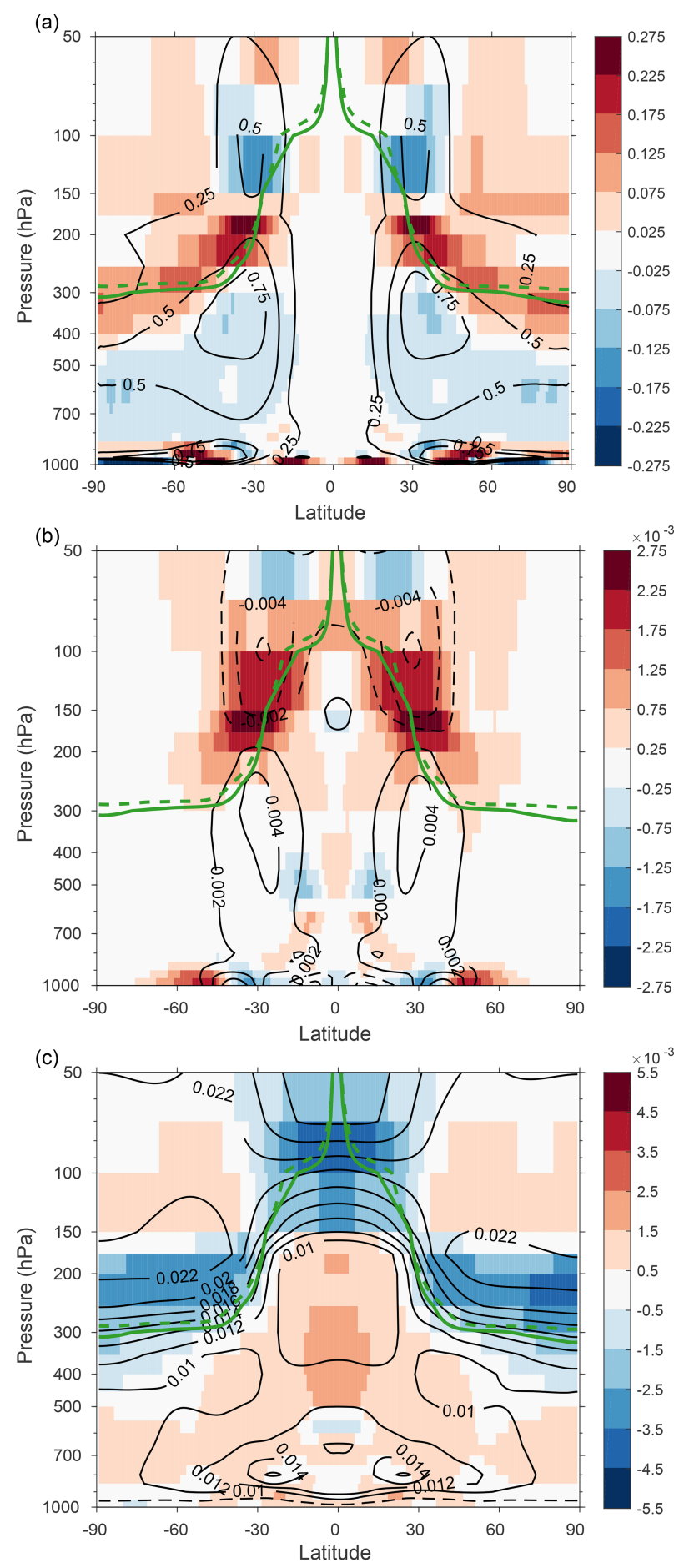

Figure 3. Zonal mean (a) maximum Eady growth rate $\left(\mathrm{d}^{-1}\right)$, (b) vertical shear of the zonal wind $\left(\mathrm{s}^{-1}\right)$, and (c) Brunt-Väisälä frequency $\left(\mathrm{s}^{-1}\right)$ averaged over 10 years of simulation. Black contours show the CNTL simulation, and shading shows the difference between the SST4 and control simulations (SST4 - CNTL). In (c) contours are every $0.002 \mathrm{~s}^{-1}$ for values greater than $0.01 \mathrm{~s}^{-1}$, and the dashed line shows the $0.005 \mathrm{~s}^{-1}$ contour. The green solid line shows the dynamic tropopause (the 2 PVU surface) in CNTL and the green dashed line in SST4. temperature gradient in the troposphere (Fig. 1a). Near the surface, there is a dipole pattern showing that the maximum in wind shear moves polewards. This is consistent with the poleward shift of the eddy-driven jet and also explains the poleward shift in the $900 \mathrm{hPa}$ Eady growth rates. The BruntVäisälä frequency increases in the troposphere, which indicates that the decrease in the Eady growth rates at $700 \mathrm{hPa}$, and at lower latitudes higher up in the troposphere, is due primarily to changes in the static stability. Near the tropopause the decrease in the stability associated with an increase in the tropopause height increases the Eady growth rate. In contrast, the decrease in the secondary maximum in the stratosphere is due to changes in the vertical wind shear.

\section{Cyclone statistics}

In this section bulk cyclone statistics are presented from both the CNTL and SST4 simulations. All cyclone tracks that meet the criteria described in Sect. 3.1 are included in this analysis, and their mean and median characteristics are summarized in Table 1. In the control simulation there are 3581 extra-tropical cyclones which have a median lifetime of $108 \mathrm{~h}(4.5 \mathrm{~d})$ and a median maximum vorticity of $5.94 \times 10^{-5} \mathrm{~s}^{-1}$ (Table 1). The uniform warming acts to decrease the total number of cyclone tracks by $3.3 \%$ but does not alter the median duration (lifetime) of extra-tropical cyclones (Table 1). The inter-annual variability in the number of cyclone tracks, quantified by calculating the number of cyclone tracks each year and then obtaining the standard deviation of these 10 values, is small (13.5 in CNTL and 10.1 in SST4) relative to the absolute decrease in the number of cyclone tracks (119). This, and a two-sided Student's $t$ test, shows that the decrease in the number of tracks is statistically significant.

Figure 4a shows histograms of maximum $850 \mathrm{hPa}$ vorticity (also referred to hereinafter as maximum intensity). There are more stronger cyclones, for example with intensities exceeding $10 \times 10^{-5} \mathrm{~s}^{-1}$, in the SST4 experiment than in the CNTL experiment. However, the mean intensity does not change considerably and there is a $3.2 \%$ decrease (equivalent to $0.19 \times 10^{-5} \mathrm{~s}^{-1}$ ) in the median maximum vorticity. This change (i.e. the signal) is very small compared to the variation between individual cyclones quantified by the standard deviation of the maximum relative vorticity of all storms $\left(2.55 \times 10^{-5} \mathrm{~s}^{-1}\right.$ in CNTL). Furthermore, the mean maximum vorticity for all cyclones occurring in each individual year can be obtained and the standard deviation of these 10 values calculated to obtain the inter-annual standard deviation of the maximum relative vorticity. For CNTL this is $0.14 \times 10^{-5}$ and $0.10 \times 10^{-5} \mathrm{~s}^{-1}$ in SST4, which are both larger than the absolute change in the mean maximum relative vorticity $\left(-0.04 \times 10^{-5} \mathrm{~s}^{-1}\right.$, Table 1$)$. A two-sided Student's $t$ test further confirms that the mean intensity does not differ in a statistically significant way between CNTL 

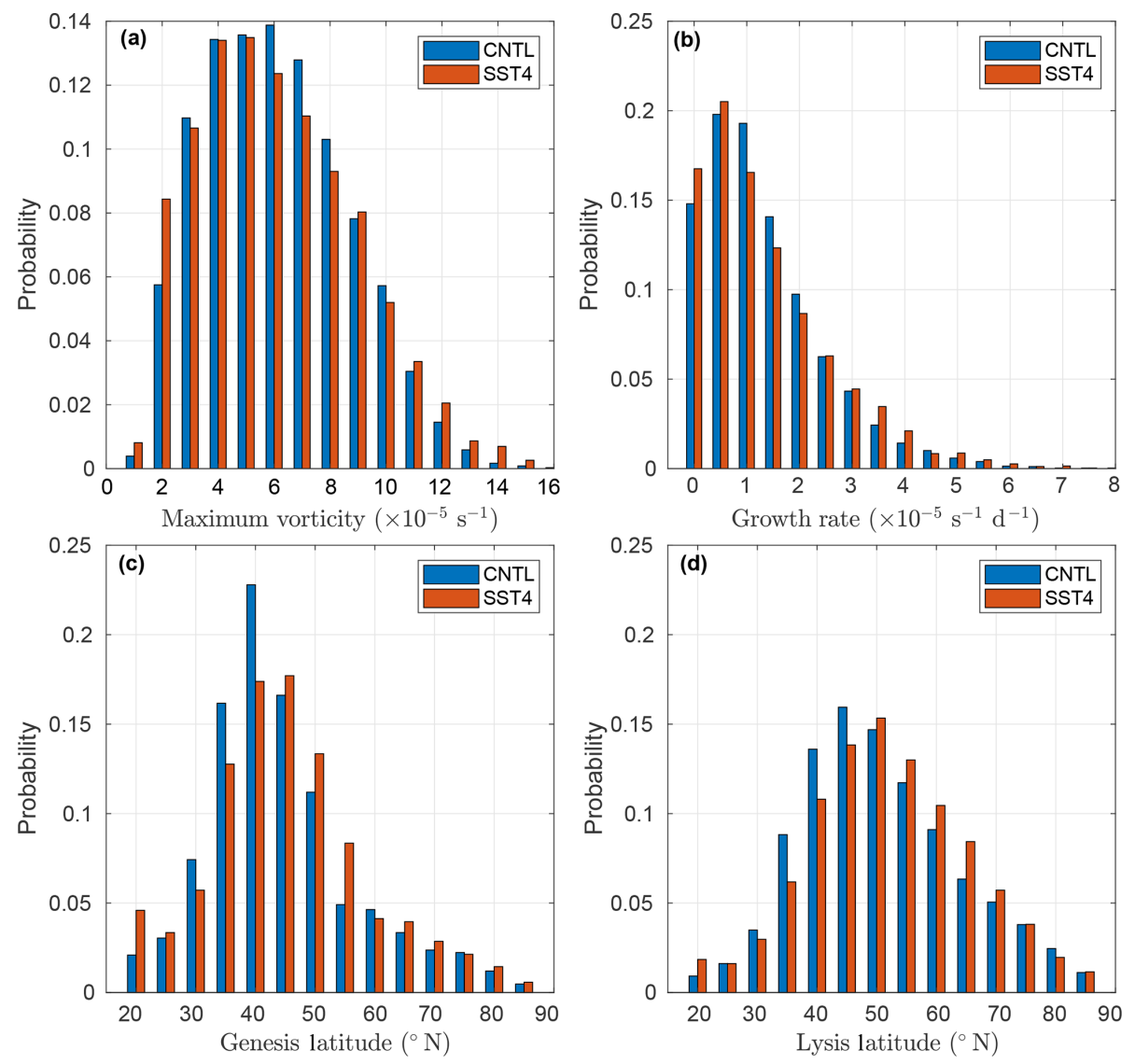

Figure 4. Normalized histograms of the extra-tropical cyclone's (a) maximum $850 \mathrm{hPa}$ relative vorticity, (b) average deepening rate between time of genesis and time of maximum vorticity, (c) genesis latitude, and (d) lysis latitude. Blue shows CNTL and red shows SST4.

and SST4, and a Wilcoxon rank-sum test shows that the median maximum intensities are not statistically significantly different between the CNTL and SST4 experiments. However, as evident from Fig. 4a, and confirmed by a one-tailed $F$ test applied to the maximum vorticity distributions, the maximum vorticity distribution in the SST4 experiment has a larger variance than in the CNTL experiment. Thus, it can be concluded that the average population of all cyclones does not change with warming but that there are more stronger and more weak cyclones in the SST4 experiment than in CNTL.

Table 1 also includes the median deepening rates of all extra-tropical cyclones. The deepening rate is the temporal rate of change of the $850 \mathrm{hPa}$ relative vorticity so that positive values indicate a strengthening, or deepening, of the extratropical cyclone. In CNTL, the relative vorticity increases by $1.31 \times 10^{-5} \mathrm{~s}^{-1}$ every $24 \mathrm{~h}$ when evaluated from genesis time to time of maximum intensity. In SST4, the corresponding value is $1.28 \times 10^{-5} \mathrm{~s}^{-1}$ per $24 \mathrm{~h}$. The change is very small in comparison to the standard deviation of the deepening rates (Table 1). Distributions of the deepening rates of all identified extra-tropical cyclones calculated between the time of genesis and time of maximum intensity are shown in Fig. 4b. A rank-sum test performed on the deepening rates between the time of genesis and time of maximum intensity confirms that the median values are not statistically different. The same test applied to the deepening rates calculated over the $24 \mathrm{~h}$ before the time of maximum intensity also shows that the control and SST4 experiments do not differ significantly. However, similar to what is found with the maximum vorticity distributions, the variance of the deepening rates is statistically significantly larger in the SST4 experiment compared to in CNTL. The lack of any notable change in the median deepening rate of all extra-tropical cyclones differs somewhat from the zonal mean calculations of the Eady growth rate (Fig. 3a), which indicate a 5\%-10\% decrease. This difference likely arises because the Eady growth rate is a measure of dry baroclinicity whereas moist processes are acting in these simulations.

Distributions of the genesis and lysis latitudes for all extratropical cyclones are shown in Fig. $4 \mathrm{c}$ and d. As hypothesized in Sect. 4, both genesis and lysis regions move poleward with warming. The median genesis region moves $2^{\circ}$ polewards from 44.2 to $46.2^{\circ} \mathrm{N}$, and the median lysis region moves poleward by $1.9^{\circ}$ from 51.4 to $53.3^{\circ} \mathrm{N}$ (Table 1 ). The interannual standard deviation of the genesis latitude is $0.27^{\circ}$ in CNTL and $0.69^{\circ}$ in SST4, suggesting that the $2^{\circ}$ poleward 
Table 1. Cyclone statistics from CNTL and SST4. Relative vorticity values have units of $\times 10^{-5} \mathrm{~s}^{-1}$. Duration (lifetime) is given in units of hours. Deepening rates (units of $\times 10^{-5} \mathrm{~s}^{-1}(24 \mathrm{~h})^{-1}$ ) are the temporal rate of change of the $850 \mathrm{hPa}$ relative vorticity. Positive deepening rate values indicate a strengthening, or deepening, of the extra-tropical cyclone. For vorticity, duration, and deepening rates, change is the relative change ((SST4 - CNTL)/CNTL) given as a percentage. For genesis and lysis latitude, change is the absolute change.

\begin{tabular}{|c|c|c|c|c|c|c|}
\hline \multirow[b]{2}{*}{ Diagnostic } & \multicolumn{3}{|c|}{ All cyclones } & \multicolumn{3}{|c|}{ Strongest 200 cyclones } \\
\hline & CNTL & SST4 & Change & CNTL & SST4 & Change \\
\hline Number of tracks/cyclones & 3581 & 3462 & $-3.3 \%$ & 200 & 200 & $0 \%$ \\
\hline Mean maximum $850 \mathrm{hPa}$ vorticity & 6.11 & 6.07 & $-0.7 \%$ & 11.55 & 11.87 & $+2.8 \%$ \\
\hline Median maximum $850 \mathrm{hPa}$ vorticity & 5.94 & 5.75 & $-3.2 \%$ & 11.24 & 11.56 & $+2.8 \%$ \\
\hline Standard deviation of maximum $850 \mathrm{hPa}$ vorticity & 2.55 & 2.80 & $+9.8 \%$ & 1.00 & 1.22 & $+22 \%$ \\
\hline Mean track duration & 132.3 & 127.8 & $-3.4 \%$ & 209.7 & 190.8 & $-9.9 \%$ \\
\hline Median track duration & 108.0 & 108.1 & $0 \%$ & 192.0 & 180.0 & $-6.25 \%$ \\
\hline Standard deviation of track duration & 77.3 & 73.5 & $-4.9 \%$ & 83.0 & 83.2 & $+0.24 \%$ \\
\hline Median deepening rate (genesis to $t=0 \mathrm{~h}$ ) & 1.31 & 1.28 & $-2.3 \%$ & 2.63 & 3.36 & $+27.7 \%$ \\
\hline Standard deviation of deepening rate (genesis to $t=0 \mathrm{~h}$ ) & 1.13 & 1.25 & $+10.6 \%$ & 1.43 & 1.65 & $+15.4 \%$ \\
\hline Median deepening rate ( $-24 \mathrm{~h}$ to $t=0 \mathrm{~h})$ & 1.42 & 1.43 & $+0.7 \%$ & 3.57 & 4.41 & $+23.5 \%$ \\
\hline Standard deviation of deepening rate $(-24 \mathrm{~h}$ to $t=0 \mathrm{~h})$ & 1.25 & 1.42 & $+13.6 \%$ & 1.53 & 1.75 & $+14.37 \%$ \\
\hline Median genesis latitude & $44.2^{\circ} \mathrm{N}$ & $46.2^{\circ} \mathrm{N}$ & $+2.0^{\circ}$ & $37.8^{\circ} \mathrm{N}$ & $38.2^{\circ} \mathrm{N}$ & $+0.4^{\circ}$ \\
\hline Median lysis latitude & $51.4^{\circ} \mathrm{N}$ & $53.3^{\circ} \mathrm{N}$ & $+1.9^{\circ}$ & $51.2^{\circ} \mathrm{N}$ & $55.0^{\circ} \mathrm{N}$ & $+3.8^{\circ}$ \\
\hline Standard deviation of genesis latitude & $12.8^{\circ}$ & $13.7^{\circ}$ & $+0.9^{\circ}$ & $8.6^{\circ}$ & $8.9^{\circ}$ & $+0.3^{\circ}$ \\
\hline Standard deviation of lysis latitude & $13.8^{\circ}$ & $14.7^{\circ}$ & $+0.9^{\circ}$ & $11.3^{\circ}$ & $11.0^{\circ}$ & $-0.3^{\circ}$ \\
\hline Median dlat (lysis-genesis latitude) & $6.2^{\circ}$ & $6.0^{\circ}$ & $-0.2^{\circ}$ & $13.7^{\circ}$ & $16.7^{\circ}$ & $+3.0^{\circ}$ \\
\hline Median dlat (max vort lat-genesis latitude) & $2.9^{\circ}$ & $2.9^{\circ}$ & $0^{\circ}$ & $9.0^{\circ}$ & $9.3^{\circ}$ & $+0.3^{\circ}$ \\
\hline $850 \mathrm{hPa}$ relative vorticity threshold for strongest 200 cyclones & - & - & - & 10.44 & 10.88 & $+4.2 \%$ \\
\hline Vorticity of the strongest cyclone & _ & - & _ & 15.55 & 16.80 & $+8.1 \%$ \\
\hline Maximum deepening (genesis to time of max) & - & - & - & 7.05 & 9.10 & $+29.0 \%$ \\
\hline
\end{tabular}

shift in genesis latitude is significant. Likewise, the interannual standard deviation of the lysis latitude is 0.52 and $0.72^{\circ}$ in CNTL and SST4 respectively and therefore also smaller than the response to warming. Two-sided Student's $t$ tests show that the mean genesis latitude differs between the CNTL and SST4 experiments at the $95 \%$ confidence level and that both the median genesis and lysis latitudes differ significantly at the 0.05 significance level. The standard deviation of both the genesis latitude $\left(12.8^{\circ}\right.$ in CNTL, Table 1$)$ and lysis latitude $\left(13.7^{\circ}\right.$ in CNTL, Table 1$)$ of all cyclones is larger than the mean change in genesis and lysis latitudes, indicating that the change is small compared to the variation between individual cyclones.

Table 1 also includes statistics for the strongest 200 extratropical cyclones in each experiment as the structure of these intense extra-tropical cyclones will be investigated in detail in Sect. 6. Firstly, the median genesis latitudes of the strongest extra-tropical cyclones are 6 to $8^{\circ}$ farther equatorward than for all extra-tropical cyclones in both the CNTL and SST4 experiments, which means that the strongest storms form in climatologically warmer and more moist environments than average-intensity storms. The more equatorward genesis region, combined with similar (CNTL) or more poleward (SST4) lysis regions, means that the strongest extra-tropical cyclones have much larger latitudinal displacements than extra-tropical cyclones do on average. Secondly, the median genesis latitude of the 200 strongest extra-tropical cyclones only moves $0.4^{\circ}$ poleward with warming, which is notably less than the $2.0^{\circ}$ poleward shift found when all extra-tropical cyclones are considered. Thirdly, deepening rates increase much more with warming for the strongest 200 extra-tropical cyclones than for all extra-tropical cyclones. Finally, the mean, median, and maximum intensity of the 200 strongest extra-tropical cyclones in the SST4 experiment are larger than in the CNTL experiment.

\section{Cyclone structure}

\subsection{Evolution of the composite cyclone in CNTL}

The cyclone composite of the strongest 200 extra-tropical cyclones in the CNTL experiment is now discussed. The temporal evolution of the composite mean cyclone in the CNTL simulation, in terms of mean sea level pressure and total column water vapour (TCWV) is shown in Fig. 5. A total of $48 \mathrm{~h}$ before the time of maximum intensity $(t=-48 \mathrm{~h}$, Fig. 5a) the composite cyclone has a closed low-pressure centre with a minimum MSLP of $978 \mathrm{hPa}$. The location of the cold and warm fronts is evident as enhanced gradients in the TCWV and the warm sector, located between the cold front and warm front, are well defined and have values of 

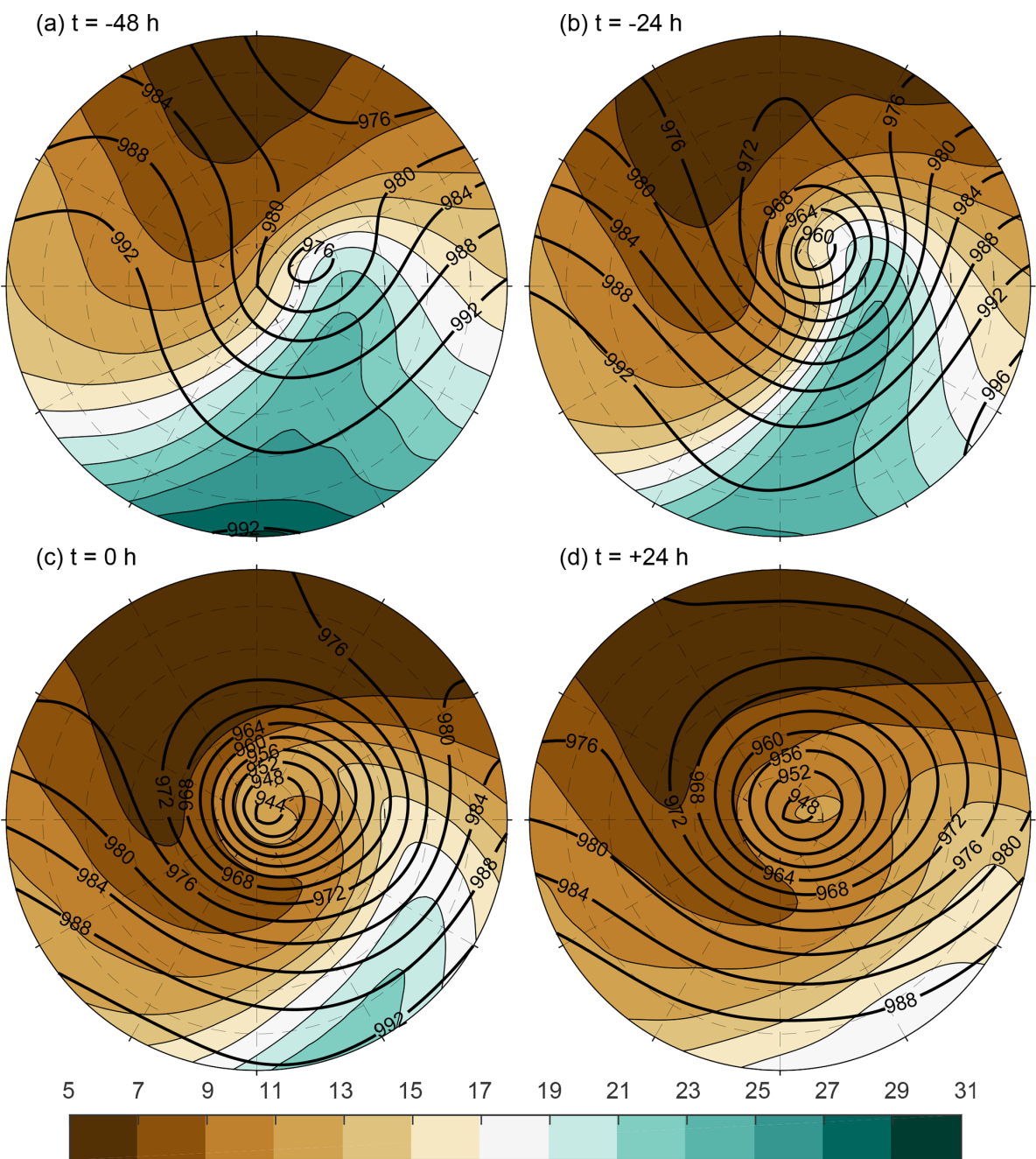

Figure 5. Composite cyclone of the strongest 200 extra-tropical cyclones in the CNTL simulation at (a) $48 \mathrm{~h}$ before time of maximum vorticity, (b) $24 \mathrm{~h}$ before time of maximum vorticity, (c) time of maximum vorticity, and (d) $24 \mathrm{~h}$ after the time of maximum vorticity. Shading shows the total column water vapour $\left(\mathrm{g} \mathrm{kg}^{-1}\right)$, and black contours show the mean sea level pressure (hPa). The plotted radius is $12^{\circ}$.

TCWV exceeding $25 \mathrm{~g} \mathrm{~kg}^{-1}$. At $24 \mathrm{~h}$ before the time of maximum intensity $(t=-24 \mathrm{~h}$, Fig. $5 \mathrm{~b})$ the low-pressure centre has become deeper (minimum MSLP of $960 \mathrm{hPa}$ ), the warm sector has become narrow and the gradients in TCWV across both the warm and cold fronts have become larger. The dry air moving cyclonically behind the cold front now extends farther south relative to the cyclone centre than it did $24 \mathrm{~h}$ earlier. By the time of maximum relative vorticity $(t=0 \mathrm{~h}$, Fig. 5c), the MSLP shows a mature, very deep cyclone which has a minimum pressure of $944 \mathrm{hPa}$. The TCWV over the whole cyclone composite area is now considerably lower than at earlier stages most likely because as the cyclones included in the composite intensify they move poleward to climatologically drier areas. The TCWV pattern also shows that the composite cyclone starts to occlude by this point $(t=0 \mathrm{~h})$ as the warm sector does not connect directly to the centre of the cyclone - instead it is displaced downstream. Finally $24 \mathrm{~h}$ after the time of maximum intensity ( $t=+24 \mathrm{~h}$, Fig. 5d), the cyclone resembles a barotropic low and has weak frontal gradients associated with it. The evolution of the composite cyclone in the CNTL experiment is, however, qualitatively very similar to real extra-tropical cyclones observed on Earth.

\subsection{Low-level potential and relative vorticity}

The response of the cyclone structure to warming is now considered primarily using changes to the mean values (i.e. SST4 - CNTL). First the temporal evolution of the lowlevel potential vorticity (PV) and the changes to this variable with warming are considered (Fig. 6). Before the composite cyclone reaches it maximum intensity (Fig. 6a and b), the maximum in the 900-700 $\mathrm{hPa}$ layer-averaged $\mathrm{PV}$ in the control simulation is poleward and downstream of the cyclone centre. By the time of maximum intensity (Fig. 6c), the maximum PV is co-located with the cyclone centre and there is 
(a) $t=-48 h$

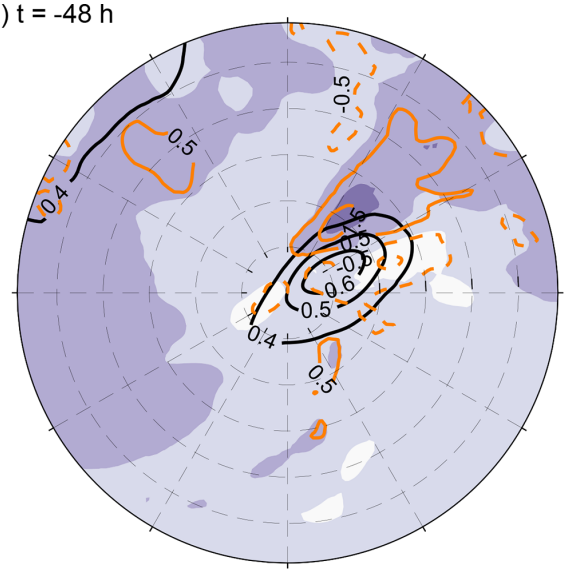

(c) $t=0 h$

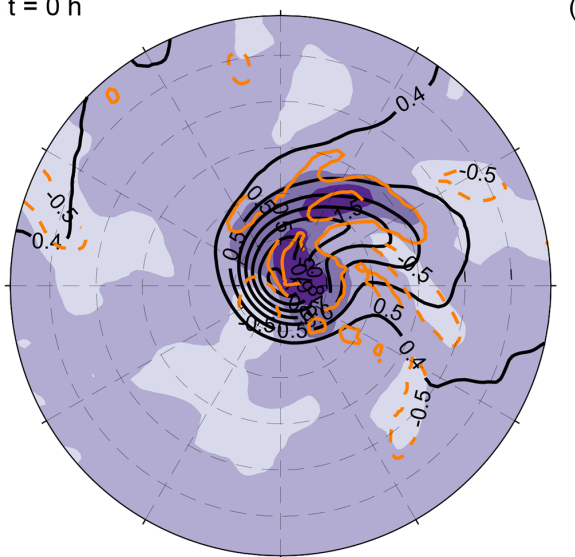

(b) $t=-24 h$

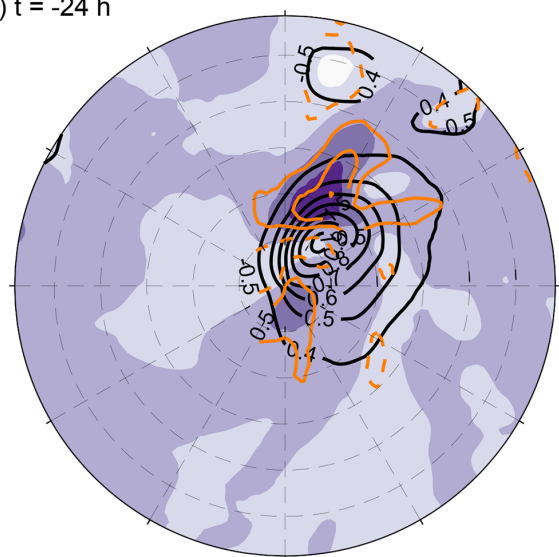

(d) $t=+24 h$

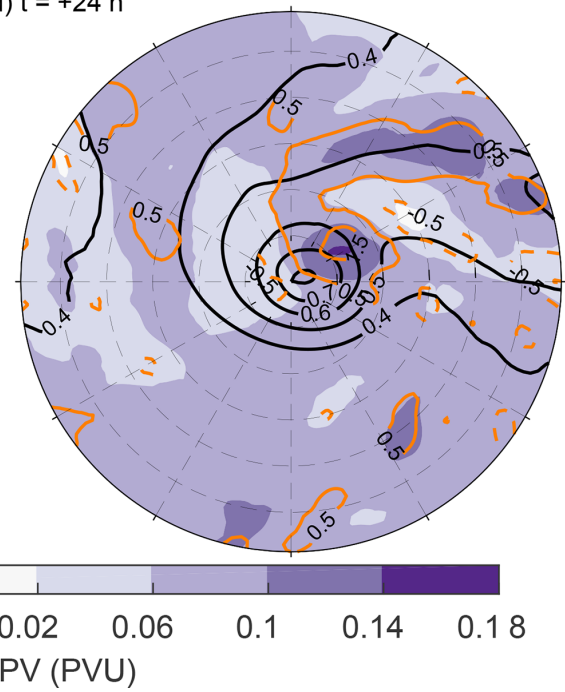

Figure 6. Composite mean of the strongest 200 extra-tropical cyclones at (a) $48 \mathrm{~h}$ before time of maximum vorticity, (b) $24 \mathrm{~h}$ before time of maximum vorticity, (c) time of maximum vorticity, and (d) $24 \mathrm{~h}$ after the time of maximum vorticity. Black contours show the $900-700 \mathrm{hPa}$ layer mean potential vorticity in CNTL (contour interval 0.1 PVU, starting at $0.4 \mathrm{PVU}$ ). Shading shows the difference in the $900-700 \mathrm{hPa}$ layer mean potential vorticity between SST4 and CNTL. Orange contours show the difference in the 850 hPa relative vorticity between SST4 and CNTL (contour interval $0.5 \times 10^{-5} \mathrm{~s}^{-1}$, the 0 contour is omitted). Solid orange contours show positive differences and dashed contours negative differences.

a secondary maximum which extends downstream of the cyclone centre and is co-located with the warm front.

At $t=-48$ and $t=-24 \mathrm{~h}$, the largest absolute increases in the $900-700 \mathrm{hPa} \mathrm{PV}$ occur poleward of the warm front location (Fig. 6a and b). This low-level PV anomaly is primarily caused by a diabatic heating maximum above this layer and therefore the poleward movement of the maximum indicates that the maximum in diabatic heating has also moved polewards with warming. The increase in PV is colocated with an increase in relative vorticity (orange contours in Fig. 6), which is consistent with an intensified cyclonic circulation beneath a region of localized heating. It can therefore be concluded that the relative vorticity associated the warm front increases with warming. At $t=0$ and $t=+24 \mathrm{~h}$ (Fig. $6 \mathrm{c}$ and d), two distinct regions of increased low-level PV are evident. The first is poleward of the warm front, as found at the earlier stages of development, and the second is almost co-located with the cyclone centre yet displaced slightly downstream. Both localized increases in lowlevel PV are also associated with increases in relative vorticity. In relative terms (not shown) the low-level potential vorticity poleward of the warm front increases by $25 \%-30 \%$, whereas near the cyclone centre the low-level PV only increases by $15 \%-20 \%$.

The response to warming also shows that almost everywhere within a $12^{\circ}$ radius of the cyclone centre, at all offset times, there is an increase in low-level PV. The absolute values of increase are smaller, mostly less than 0.1 PVU, but in relative terms the increase is similar in magnitude to that found near the warm front and cyclone centre. Away from the cyclone centre, where there is no significant relative vorticity, this increase in low-level PV is primarily caused by an 
(a) $t=-48 h$

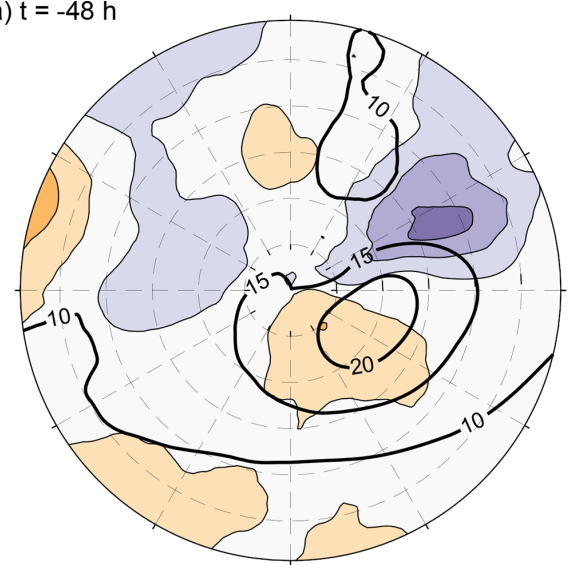

(c) $t=0 h$

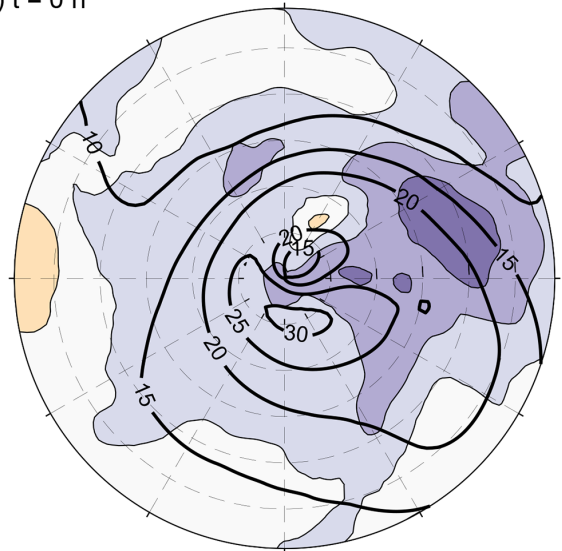

(b) $t=-24 h$

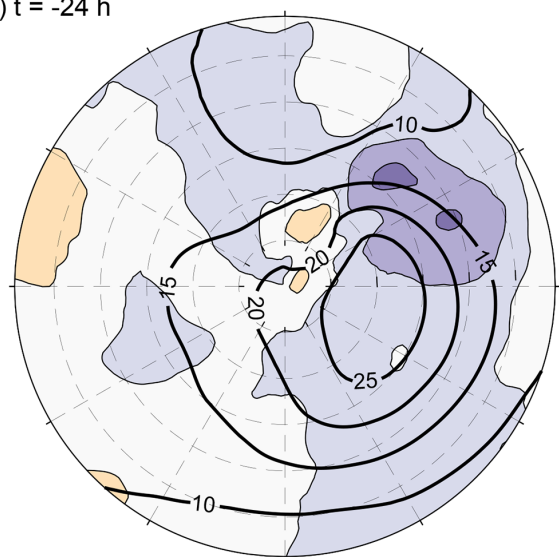

(d) $t=24 h$

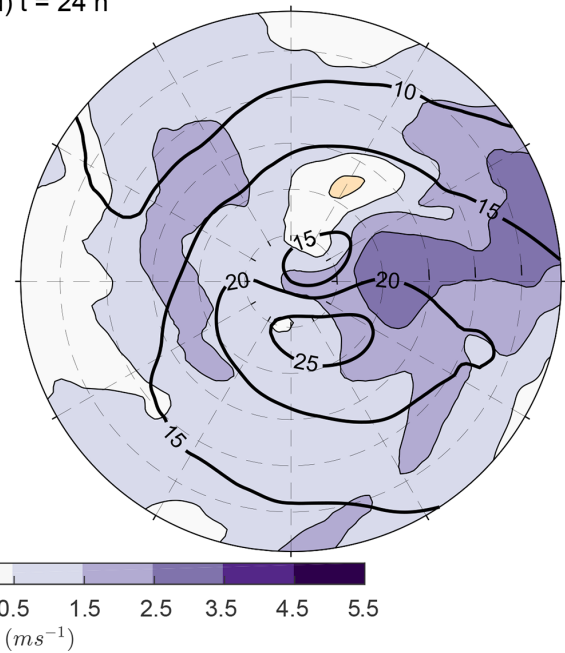

Figure 7. Composite mean of the $900 \mathrm{hPa}$ wind speed of the strongest 200 extra-tropical cyclones in the CNTL simulation (black contours, every $5 \mathrm{~m} \mathrm{~s}^{-1}$ ) and the difference between SST4 and CNTL (shading) at (a) $48 \mathrm{~h}$ before time of maximum intensity, (b) $24 \mathrm{~h}$ before time of maximum intensity, (c) time of maximum intensity, and (d) $24 \mathrm{~h}$ after the time of maximum intensity.

increase in stratification. However, given that the cyclones are more poleward in the SST4 experiment, the increase in planetary vorticity also plays a small role.

\subsection{Low-level wind speed}

Figure 6 highlights that the relative vorticity increases with warming at all offset times. Associated with this increase in relative vorticity is an increase in low-level horizontal wind speeds. In the composite from the CNTL experiment, at $t=-48$ and $t=-24 \mathrm{~h}$ (Fig. 7a and b), the strongest $900 \mathrm{hPa}$ wind speeds exceed 20 and $25 \mathrm{~m} \mathrm{~s}^{-1}$ respectively and occur in the warm sector. At the time of maximum intensity (Fig. 7c), the strongest $900 \mathrm{hPa}$ winds in CNTL are located equatorward of the cyclone centre, behind the cold front in a very dry area, and exceed $30 \mathrm{~m} \mathrm{~s}^{-1}$. By $t=24 \mathrm{~h}$ (Fig. 7d) the wind speeds have started to weaken. At $t=-48 \mathrm{~h}$, a dipole structure in the change in wind speed due to warming is evident, indicating that the maximum wind speeds move poleward and downstream relative to those in CNTL
(Fig. 7a). However, the positive values are greater in magnitude than the negative values, thus indicating an overall increase in wind speed. At $t=-24,0$, and $24 \mathrm{~h}$ (Fig. 7b-d) the $900 \mathrm{hPa}$ winds speeds of the composite cyclone increase with warming by $\sim 1.5 \mathrm{~m} \mathrm{~s}^{-1}$ in a large area surrounding the cyclone and by up to $3.5 \mathrm{~m} \mathrm{~s}^{-1}$ in the warm front area. Consequently, the size of the area affected by wind speeds over a fixed threshold value increases, indicating greater wind risk in warmer climates. As the increase at all offset times is not co-located with maximum wind speed in CNTL, this suggests that the spatial structure of the composite extra-tropical cyclone changes with warming.

\subsection{Total column water vapour}

The response of the TCWV is now considered (Fig. 8). The uniform warming leads to an increase in TCWV everywhere in the cyclone composite at all offset times. The largest absolute increases occur at $t=-48$ and $t=-24 \mathrm{~h}$ (Fig. 8a and $b)$. At both of these offset times, the largest absolute in- 
(a) $\mathrm{t}=-48 \mathrm{~h}$

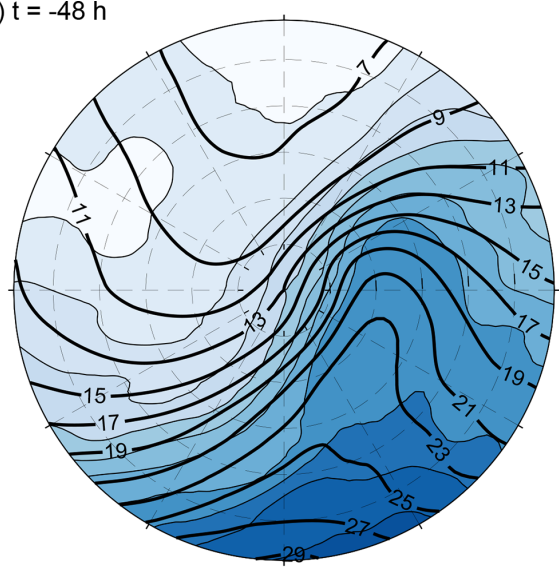

(c) $t=0 h$

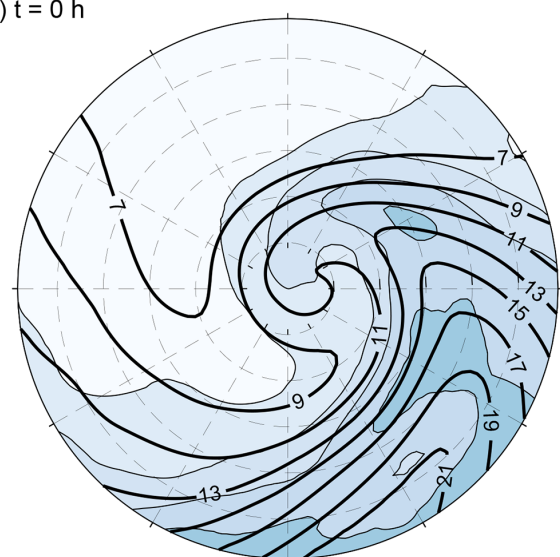

(b) $t=-24 h$

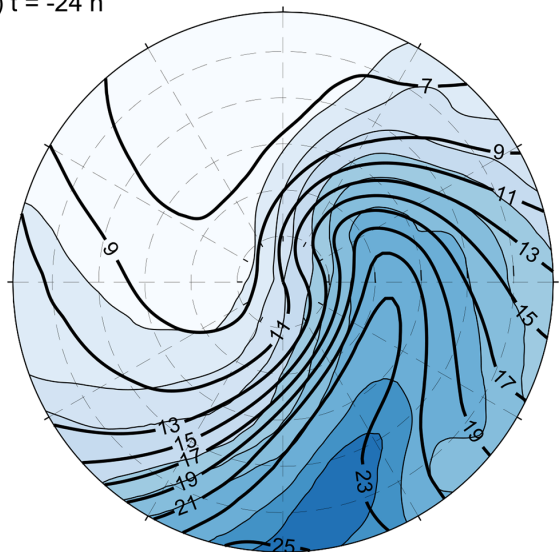

(d) $\mathrm{t}=+24 \mathrm{~h}$

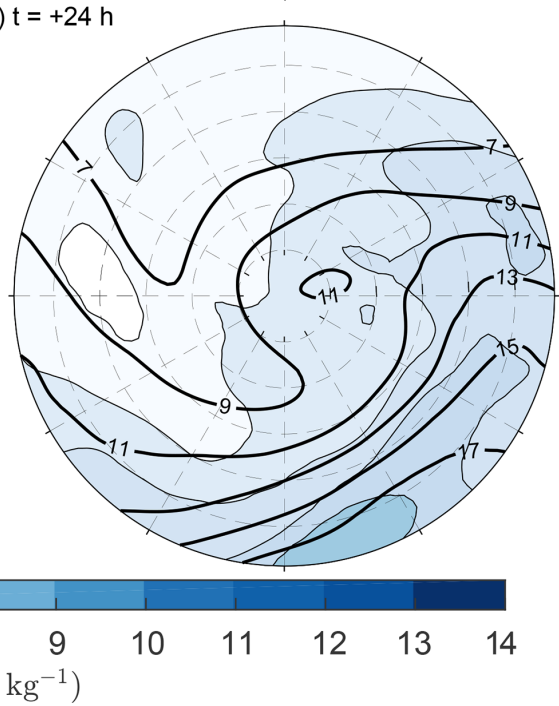

Figure 8. Composite mean of the total column water vapour (TCWV) of the strongest 200 extra-tropical cyclones in the CNTL simulation (black contours, every $2 \mathrm{~g} \mathrm{~kg}^{-1}$ ) and the difference between SST4 and CNTL (shading) at (a) $48 \mathrm{~h}$ before time of maximum intensity, (b) $24 \mathrm{~h}$ before time of maximum intensity, (c) time of maximum intensity, and (d) $24 \mathrm{~h}$ after the time of maximum intensity.

crease occurs in the warm sector where the mean values are largest in the control simulation. In terms of percentage increase (not shown), at $t=-24 \mathrm{~h}$, the TCWV increases the least, approximately $25 \%$, in the cold sector upstream of the cyclone centre and the most ahead of the warm front where the increase exceeds $50 \%$. At the time of maximum intensity (Fig. 8c), absolute increases of up to $6 \mathrm{~g} \mathrm{~kg}^{-1}$ are still evident in the warm sector and in a localized region northeast of the cyclone centre, whereas at $t=+24 \mathrm{~h}$ (Fig. 8d) increases of this magnitude are constrained to the most southern part of the cyclone composite. The composites also show the meridional moisture gradient across the composite cyclone increases notably with warming since the absolute increase is much larger in the most equatorward regions (e.g. $12 \mathrm{~g} \mathrm{~kg}^{-1}$ at $t=-48 \mathrm{~h}$ ) than in the most poleward regions (e.g. an increase of $2 \mathrm{~g} \mathrm{~kg}^{-1}$ ).

\subsection{Precipitation}

The response of the total, convective, and large-scale precipitation to warming is now considered. Composites of total, large-scale, and convective precipitation are shown in Fig. 9 valid 48, 24, and $0 \mathrm{~h}$ before the time of maximum intensity. Precipitation is calculated as the $6 \mathrm{~h}$ accumulated value centred on the valid time in units of millimetres per $6 \mathrm{~h}$. In the CNTL simulation the maximum total precipitation is downstream and poleward of the cyclone centre at all offset times. At $t=-48 \mathrm{~h}$, the total precipitation has maximum values of $6 \mathrm{~mm}(6 \mathrm{~h})^{-1}$ and is mainly located in the warm sector of the cyclone and near the warm front (Fig. 9a). At $t=-24 \mathrm{~h}$, the total precipitation in the CNTL simulation is slightly larger, covers a greater area, and has a more distinct comma shape than $24 \mathrm{~h}$ earlier (Fig. 9d). Also at this time, large values of total precipitation are evident along the cold front to the south of the cyclone centre. By the time of maximum intensity the total precipitation in the CNTL experiment has started to de- 

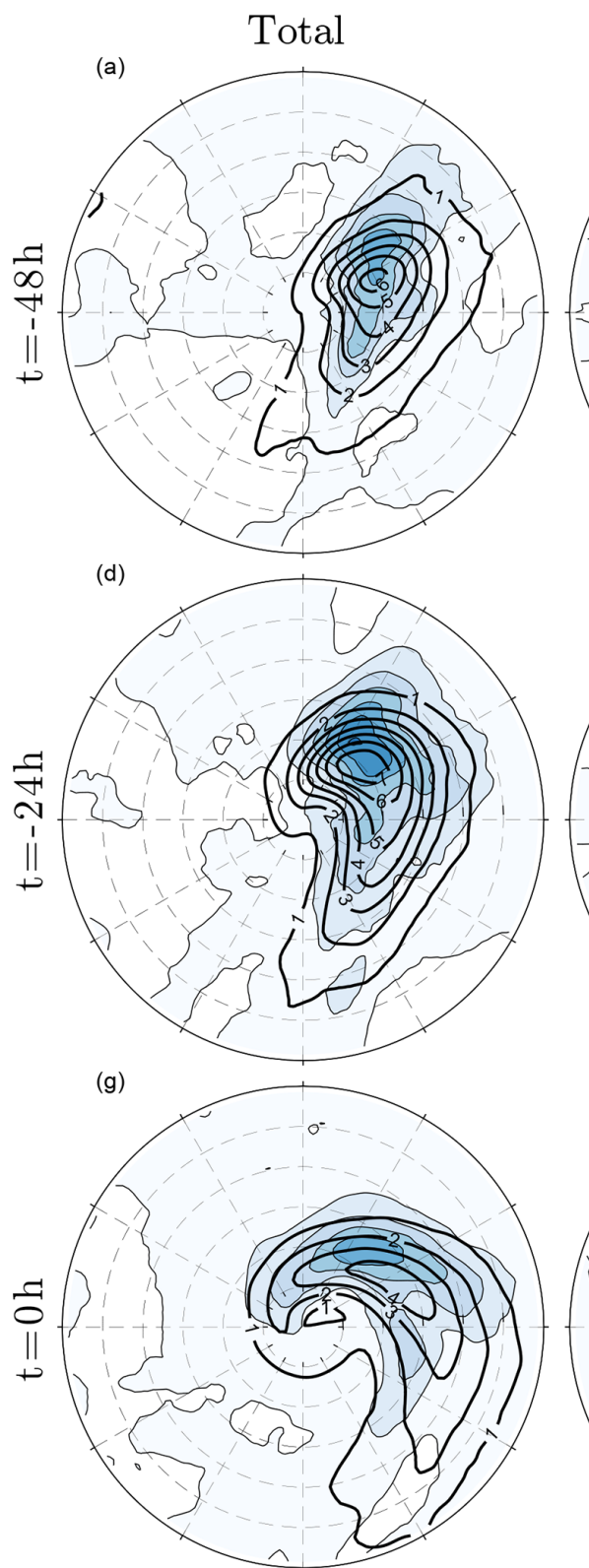

(h)
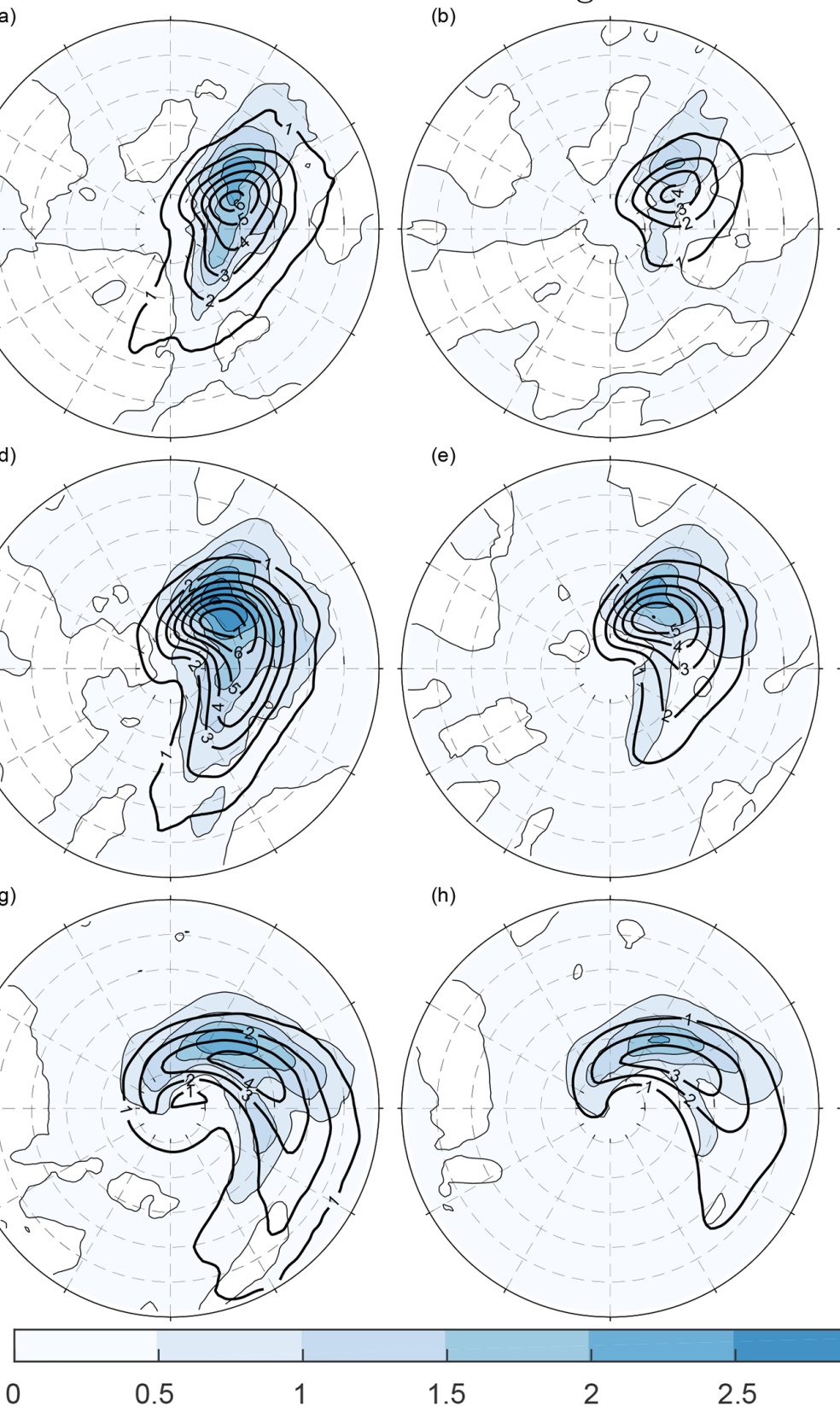

(e)

Large-scale
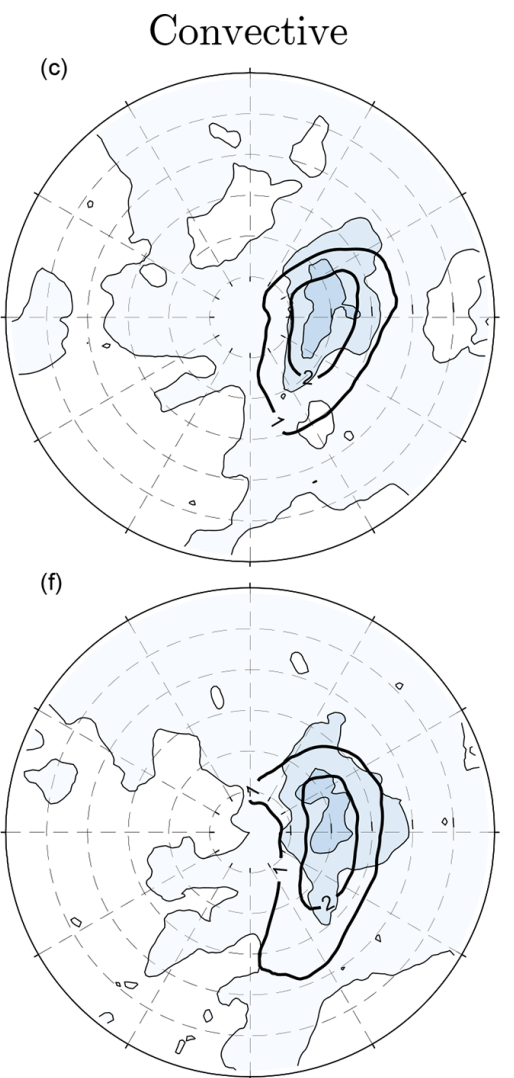

(i)

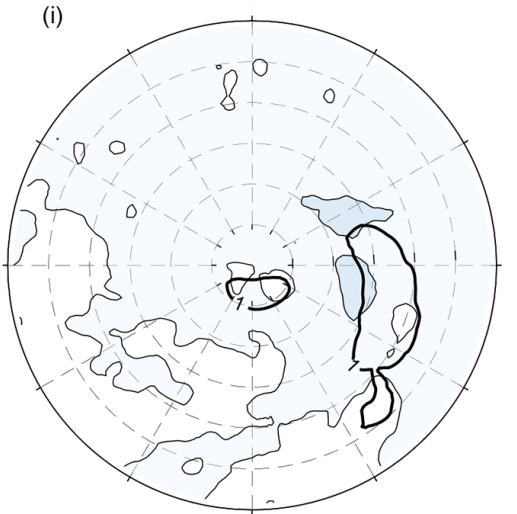

$\begin{array}{ll}1.5 & 2.5 \\ \text { Precipitation }(\mathrm{mm} \text { per } & 6 \mathrm{~h})\end{array}$

Figure 9. Composites of total precipitation (a, d, $\mathbf{g})$, large-scale precipitation $(\mathbf{b}, \mathbf{e}, \mathbf{h})$, and convective precipitation (c, f, i) in the CNTL simulation (black contours) and the difference between SST4 and control (shading). Panels (a)-(c) are valid $48 \mathrm{~h}$ before the time of maximum intensity, panels (d)-(f) are valid $24 \mathrm{~h}$ before the time of maximum intensity, and panels (g)-(i) are valid at the time of maximum intensity. All composites are of the strongest 200 extra-tropical cyclones in each experiment.

crease, with maximum values of $4 \mathrm{~mm}(6 \mathrm{~h})^{-1}$, and the location of the precipitation has rotated cyclonically around the cyclone centre (Fig. 9g).

The response to warming of the total precipitation is a large absolute and relative increase at all offset times. The maximum absolute increases are of the order of 2.5, 3.5, and
$2.0 \mathrm{~mm}(6 \mathrm{~h})^{-1}$ at $t=-48, t=-24 \mathrm{~h}$, and the time of maximum vorticity $(t=0 \mathrm{~h})$ respectively. These values correspond to relative increases of up to almost $50 \%$. The maximum increase in the total precipitation is not co-located with the maximum in the CNTL simulation, indicating that the spatial structure of the composite cyclone has changed with 
warming. The largest increases in total precipitation occur in the warm front region, poleward and downstream of the maximum in the CNTL simulation at all offset times. This spatial change is largely similar to that found when the 900$700 \mathrm{hPa}$ potential vorticity response to warming was considered (Fig. 6). This is consistent in the sense that more precipitation, and particularly more condensation, results in more latent heating and thus a stronger positive $\mathrm{PV}$ anomaly beneath the localized heating. However at $t=0 \mathrm{~h}$, it is interesting to note that while there is only one localized area where precipitation increases in SST4 compared to in CNTL (Fig. 9g), which is ahead of the warm front, there are two regions where the low-level PV increases (Fig. 6c). One of these regions is co-located with the increase in precipitation but the second region is closer to the cyclone centre. While this may be due to the larger mean relative vorticity of the strongest $200 \mathrm{cy}$ clones in SST4 compared to CNTL (Table 1), it is also possible that this second area of enhanced PV may be due to enhanced advection by the cold conveyor belt of PV produced diabatically in the warm front region, beneath the ascending warm conveyor belt. Schemm and Wernli (2014) noted such a mechanism in their study linking warm and cold conveyor belts.

The contribution of the large-scale stratiform precipitation calculated from the cloud scheme and the convective precipitation produced by the convection scheme to the total precipitation is now considered. In CNTL, the large-scale precipitation (Fig. 9b, e, and h) contributes more to the total precipitation than the convective precipitation (Fig. 9c, f, and i), particularly at $t=-24 \mathrm{~h}$ and the time of maximum intensity. However, the convective precipitation is larger and of equal magnitude to the large-scale precipitation in the more equatorward parts of the warm sector of the CNTL composite cyclone where the temperature and moisture content are higher. The large-scale precipitation increases in SST4 compared to CNTL in the warm frontal region, poleward of the maximum in the CNTL simulation, at all offset times. This spatial shift is very similar to that observed for the total precipitation, meaning that the resolved precipitation is leading to the poleward shift in the total precipitation with warming. However, the large-scale precipitation also has a smaller increase $(1-1.5 \mathrm{~mm}$ per $6 \mathrm{~h}$ ) in a narrow band along the cold front, upstream of the maximum in the control simulation, which is most evident at $t=-24 \mathrm{~h}$. In contrast, the convective precipitation, which increases by almost $50 \%$, has the largest increases co-located with the maximum in the control simulation, meaning that the position of convective precipitation relative to the cyclone centre does not change with warming.

\subsection{Vertical velocity}

The mean cyclone composite of vertical velocity at $700 \mathrm{hPa}$ (given in pressure coordinates, $\mathrm{Pa} \mathrm{s}^{-1}$ ) obtained directly from the model simulations and the response to warming is shown in Fig. 10a, c, and e. In the CNTL simulation at $t=-48$ and $t=-24 \mathrm{~h}$, there is large coherent area of ascent downstream of the cyclone centre largely co-located with the warm sector indicative of the warm conveyor belt, an ascending airstream associated with extra-tropical cyclones. At $t=0 \mathrm{~h}$ (Fig. 10e), the area of ascent is still maximized in the warm sector region but is further downstream relative to the cyclone centre than at earlier times. The ascent at $t=0 \mathrm{~h}$ has also started to wrap cyclonically around the poleward and upstream side of the cyclone, meaning that the cyclone has formed a bent-back warm front and likely has started to occlude. The absolute magnitude of the largest values of ascent occur at $t=-24 \mathrm{~h}$ and exceed $0.6 \mathrm{~Pa} \mathrm{~s}^{-1}$ (Fig. 10c), approximately $6 \mathrm{~cm} \mathrm{~s}^{-1}$. A region of weak descent is evident behind the cold front in the drier air mass at all offset times.

Uniform warming changes the vertical motion in a complex manner. The largest increases in ascent are not colocated with the strongest ascent in the CNTL simulation and instead occur poleward and downstream of the maximum. This pattern is present at all offset times and suggests that the warm front and the warm conveyor belt are located farther poleward relative to the cyclone centre in the SST4 simulation. This is consistent with the response of the total and large-scale precipitation, and the low-level potential vorticity, which also showed a poleward shift in the warm frontal region. A tri-pole structure is also evident in Fig. 10a, c, and e, which show that the area of ascent either weakens in the centre and broadens with warming or the ascent associated with the warm and cold fronts becomes more spatially separate with warming. The first of these two options will prove to be correct.

To further understand the spatial pattern of the response of the vertical velocity to warming, the contribution to the total vertical velocity from vorticity advection, thermal advection, and diabatic processes as diagnosed by the omega equation (Eq. 1) is examined. The sum of these three terms (Fig. 10b, d, and f) at $700 \mathrm{hPa}$ is first compared to the total model calculated vertical motion (Fig. 10a, c, and e). At $t=-48 \mathrm{~h}$, the diagnosed ascent in CNTL is slightly weaker than the model-calculated (i.e. direct from OpenIFS) ascent, particularly in the cold front region. The response of the diagnosed vertical motion to warming is however spatially similar to that of the model-calculated vertical motion. At $t=-24 \mathrm{~h}$, the diagnosed ascent is slightly stronger than the model-calculated ascent and covers a larger area, especially in the zonal direction. In addition, the descent diagnosed from Eq. (1) covers a smaller area than descent in the modelcalculated vertical motion field. Similar differences between the model-calculated and diagnosed vertical motion occur at $t=0 \mathrm{~h}$. There is, however, broad agreement between the model-calculated vertical motion and the vertical motion diagnosed using Eq. (1) in CNTL at all offset times, and the response to warming in the diagnosed vertical motion field is very similar to that in the model-calculated field. Thus, the individual contributions to the diagnosed ascent will provide 

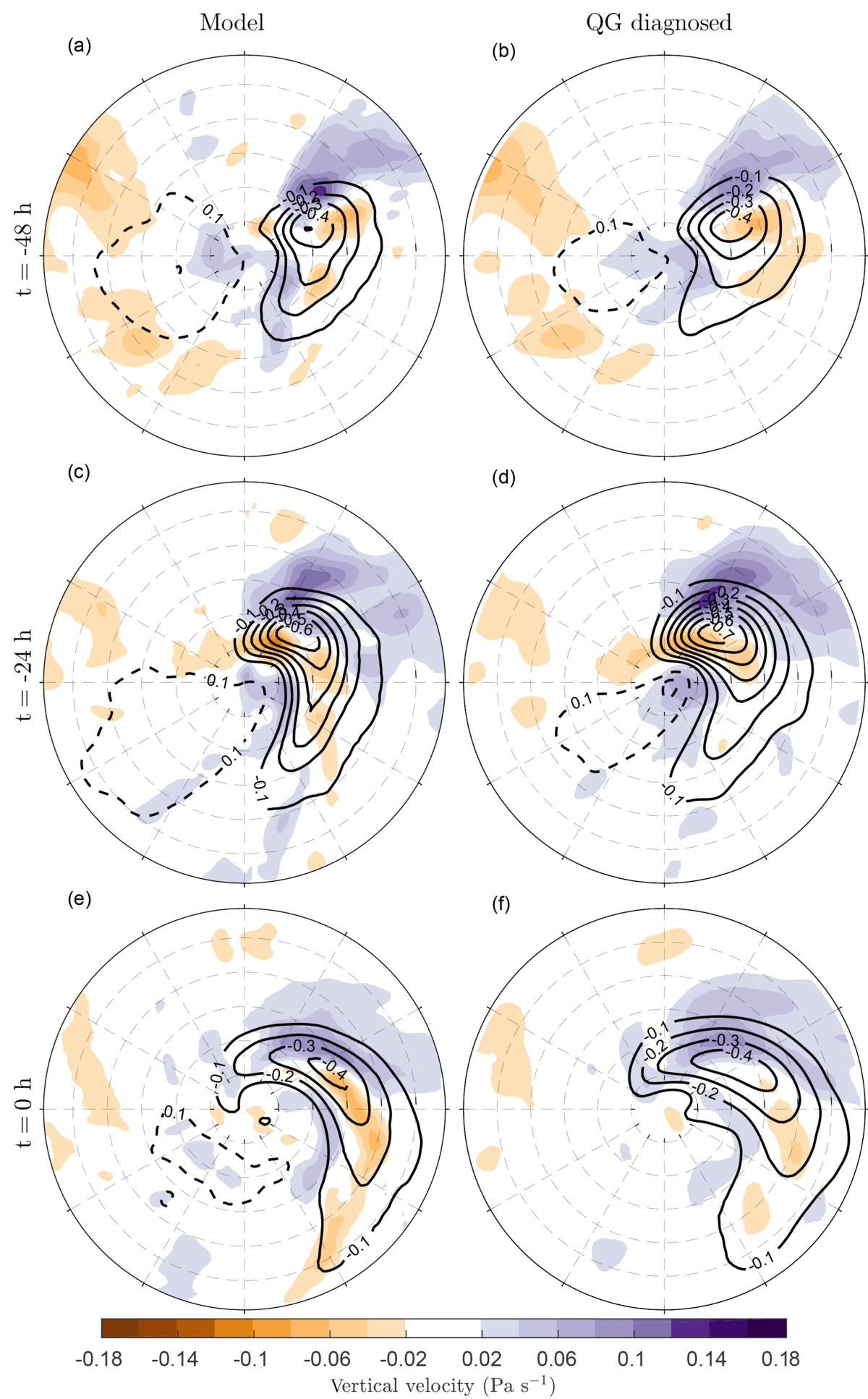

Figure 10. Composite mean and change in $700 \mathrm{hPa}$ vertical velocity in pressure coordinates for (a, c, e) total model calculated vertical velocity and (b, d, f) vertical velocity calculated from the modified quasi-geostrophic omega equation (Eq. 1). Panels (a) and (b) are valid $48 \mathrm{~h}$ before the time of maximum intensity, panels (c) and (d) are valid $24 \mathrm{~h}$ before the time of maximum intensity, and panels (e) and (f) are valid at the time of maximum intensity. All composites are of the strongest 200 extra-tropical cyclones in each experiment. 

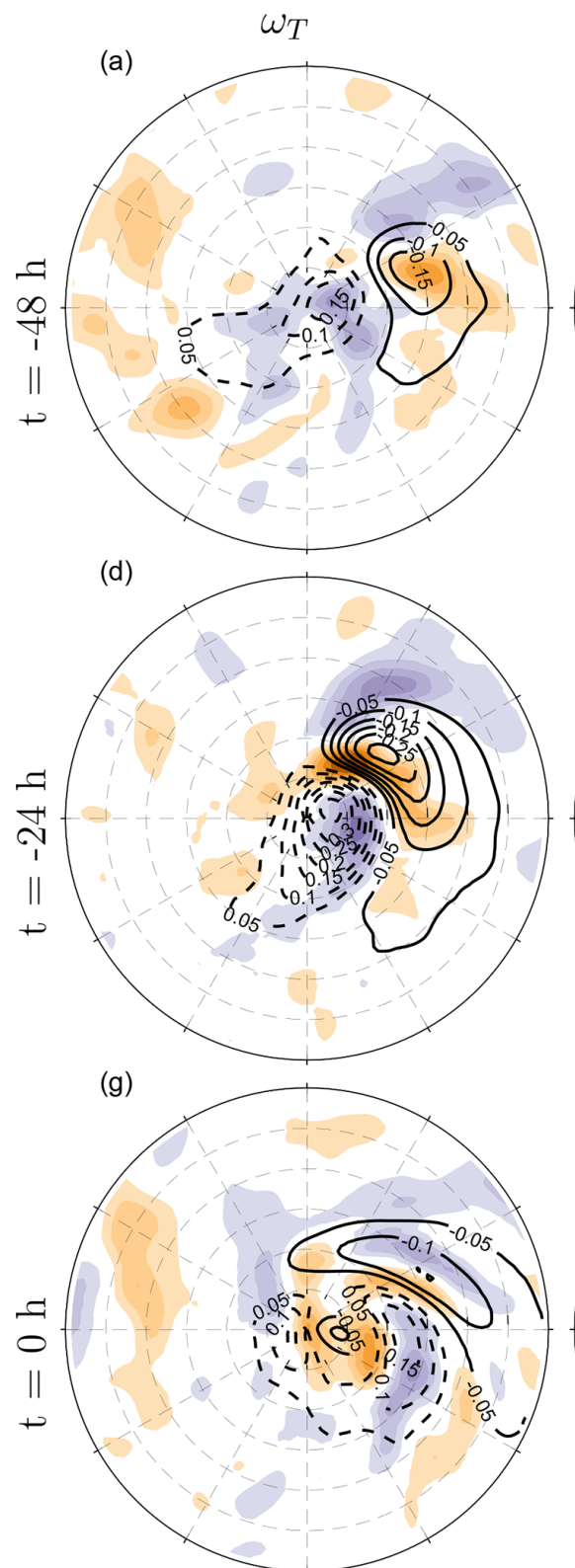

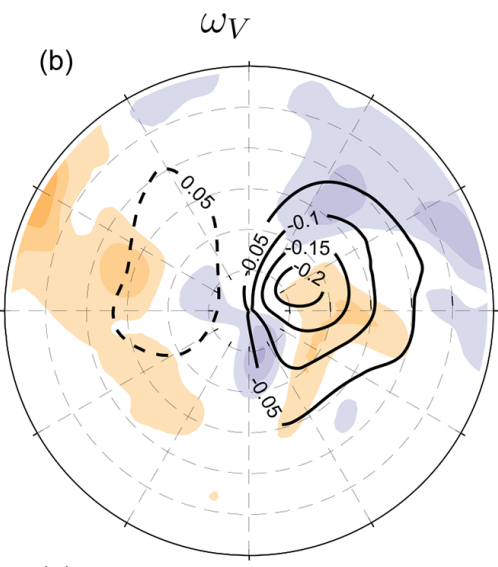

(e)

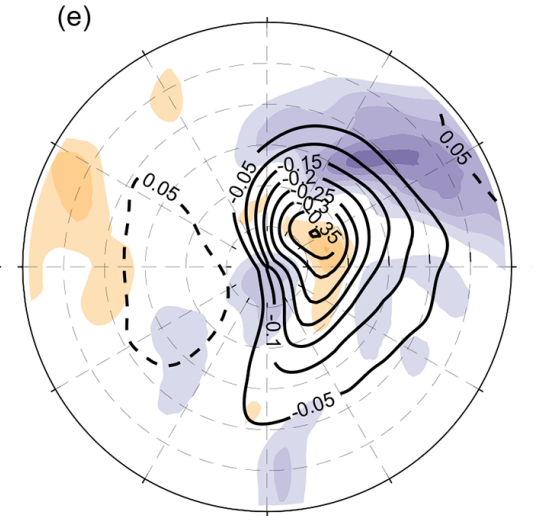

(h)

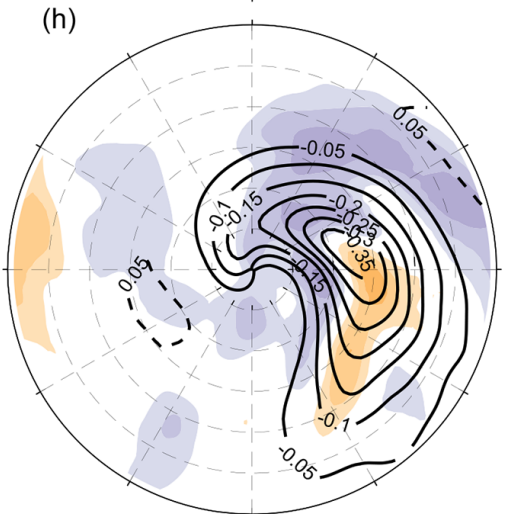

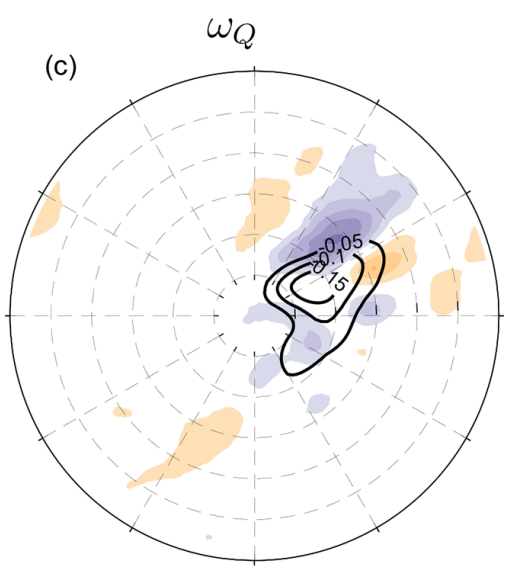

(f)

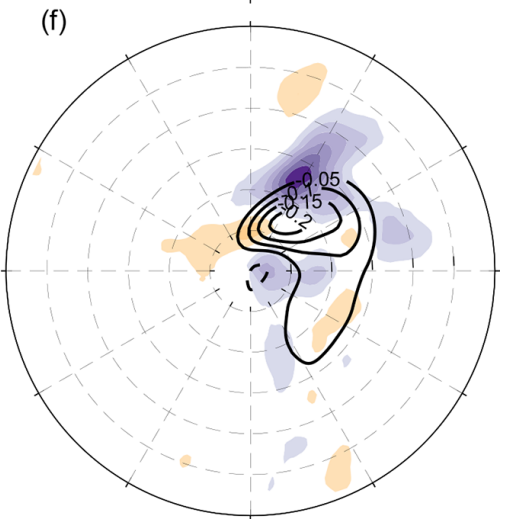

(i)

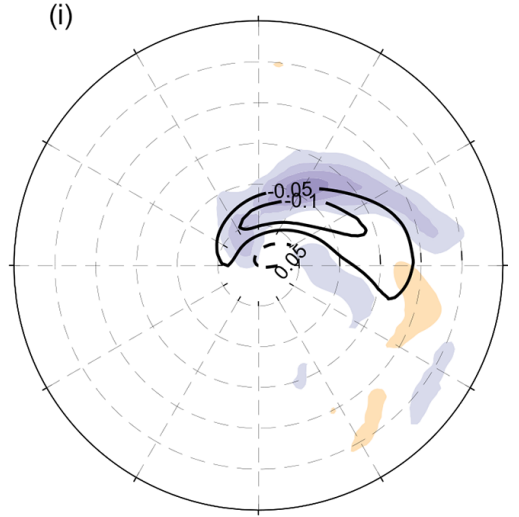

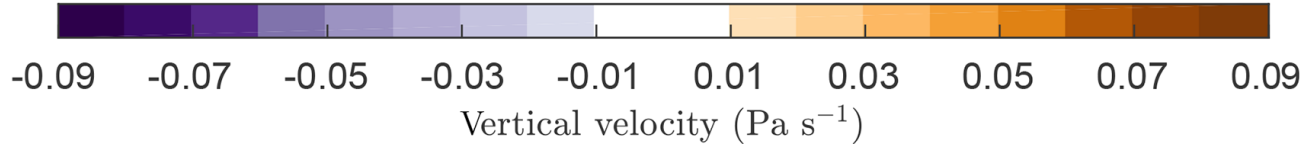

Figure 11. Composite mean and change in $700 \mathrm{hPa}$ vertical velocity in pressure coordinates due to thermal advection $\left(\omega_{\mathrm{T}}, \mathbf{a}, \mathbf{d}, \mathbf{g}\right)$, due to vorticity advection $\left(\omega_{\mathrm{V}}, \mathbf{b}, \mathbf{e}, \mathbf{h}\right)$, and due to diabatic heating $\left(\omega_{Q}, \mathbf{c}, \mathbf{f}, \mathbf{i}\right)$. Panels (a)-(c) are valid $48 \mathrm{~h}$ before the time of maximum intensity, panels (d)-(f) are valid $24 \mathrm{~h}$ before the time of maximum intensity, and panels (g)-(i) are valid at the time of maximum intensity. Contours show the control values and shading the difference (SST4 - CNTL). All composites are of the strongest 200 extra-tropical cyclones in each experiment.

reliable estimates of how different physical processes influence the total vertical motion.

Figure 11 shows the contribution to vertical velocity from thermal advection, vorticity advection, and diabatic heating at three offset times in the CNTL experiment. The maximum values of ascent attributed to the different forcing mechanisms in the composite mean cyclones are also shown in Table 2. In the CNTL composite cyclone thermal advection leads to ascent in the warm sector downstream of the cyclone centre and descent behind the cold front (Fig. 11a, d, and g). 
Table 2. Maximum values of ascent $\left(\mathrm{Pa} \mathrm{s}^{-1}\right)$ at $700 \mathrm{hPa}$ directly from the model $(\omega)$ and attributed to vorticity advection $\left(\omega_{\mathrm{V}}\right)$, thermal advection $\left(\omega_{\mathrm{T}}\right)$, and diabatic heating $\left(\omega_{Q}\right)$ in the CNTL composite mean and the SST4 composite mean at different offset times.

\begin{tabular}{|c|c|c|c|c|c|c|c|c|}
\hline \multirow[b]{2}{*}{ Time (h) } & \multicolumn{4}{|c|}{ CNTL } & \multicolumn{4}{|c|}{ SST4 } \\
\hline & $\omega$ & $\omega_{\mathrm{V}}$ & $\omega_{\mathrm{T}}$ & $\omega_{Q}$ & $\omega$ & $\omega_{\mathrm{V}}$ & $\omega_{\mathrm{T}}$ & $\omega_{Q}$ \\
\hline-48 & -0.5016 & -0.2279 & -0.1808 & -0.195 & -0.5238 & -0.2162 & -0.1423 & -0.193 \\
\hline-24 & -0.6722 & -0.401 & -0.3179 & -0.2311 & -0.6965 & -0.3816 & -0.3013 & -0.2339 \\
\hline 0 & -0.4387 & -0.3889 & -0.1517 & -0.1352 & -0.4565 & -0.3761 & -0.1681 & -0.1512 \\
\hline 24 & -0.1572 & -0.1701 & -0.0601 & -0.0371 & -0.1674 & -0.1851 & -0.054 & -0.0358 \\
\hline
\end{tabular}

Ascent and descent due to thermal advection reach a maximum $24 \mathrm{~h}$ before the time of maximum intensity (Table 2), and as the composite cyclone evolves in time the region of ascent wraps cyclonically around the poleward side of the cyclone centre. Vorticity advection (Fig. 11b, e, and h) leads to stronger ascent at all offset times compared to either thermal advection or diabatic heating in the CNTL composite cyclone (Fig. 11, Table 2). At all offset times the ascent attributed to vorticity advection covers a large area and is located downstream of the cyclone centre. As the cyclone evolves, the area of maximum ascent moves further away from the cyclone centre and a region of weak ascent wraps cyclonically around the cyclone centre. This cyclonic behaviour, indicative of life cycle 2 (LC2, Thorncroft et al., 1993) cyclone development, occurs as the cyclones are located on the poleward side of the jet at all offset times considered here. The contribution to vertical motion from the diabatic heating term is shown in Fig. 11c, f, and i. Ascent related to diabatic heating is constrained to a smaller area than ascent due to either vorticity or thermal advection and is located in the poleward parts of the warm conveyor belt. The maximum values of ascent related to diabatic heating at $t=-24 \mathrm{~h}$ and the time of maximum intensity are also weaker than those due to either thermal advection or vorticity advection in both the CNTL and SST4 experiments (Table 2). This shows that at $700 \mathrm{hPa}$ diabatic heating has a smaller impact on the cyclone's vertical motion than the dynamical terms.

The response of vertical motion due to the different forcing mechanisms to warming is also shown in Fig. 11 by the shading. At $t=-48 \mathrm{~h}$, ascent due to thermal advection weakens slightly with warming (orange shading co-located with the maximum ascent in CNTL in Fig. 11a and Table 2), and the descent associated with cold-air advection is also weaker in SST4 than in the CNTL. The ascent due to warmair advection in the warm sector is slightly more poleward in SST4 compared to CNTL. At $t=-24 \mathrm{~h}$, the region of ascent due to thermal advection in SST4 has moved poleward and downstream relative to that in the CNTL. This is evident in Fig. 11d as the positive difference values (weaker ascent, orange shading) between the cyclone centre and the maximum ascent in the CNTL and as negative difference values (stronger ascent, purple shading) poleward of the maximum ascent in CNTL. This illustrates that at both $t=-48$ and $t=-24 \mathrm{~h}$ the warm front is more poleward and extends further downstream away from the cyclone centre in SST4 compared to in CNTL. A similar but weaker pattern also remains at the time of maximum intensity (Fig. 11g).

The response of vertical motion due to vorticity advection at $700 \mathrm{hPa}$ has a similar spatial pattern at all offset times considered but is most pronounced in magnitude at $t=-24 \mathrm{~h}$ and at the time of maximum intensity. The most notable feature is that ascent due to vorticity advection in SST4 covers a greater area compared to in CNTL and that the ascent expands polewards and downstream of the cyclone centre. The second notable feature is that at $700 \mathrm{hPa}$ the maximum ascent due to vorticity advection decreases with warming (Fig. 11b, e, and h and Table 2). This indicates that ascent due to positive vorticity advection downstream of the cyclone centre weakens in magnitude but becomes more spatially extensive.

To further understand the change in ascent due to vorticity advection, the $500 \mathrm{hPa}$ geopotential height fields are considered. To compare the $500 \mathrm{hPa}$ geopotential height in the SST4 and CNTL composite cyclones, first the composite cyclone mean (weighted by grid area) at each offset time was subtracted to generate maps of the cyclone relative $500 \mathrm{hPa}$ geopotential height anomaly. This was necessary as in SST4 the $500 \mathrm{hPa}$ heights are higher simply due to the warmer atmosphere, which makes a comparison of the shape and extent of the upper level trough difficult. Figure 12 shows the differences in these anomalies. At $t=-48 \mathrm{~h}$ (Fig. 12a) the negative anomaly to the south of the cyclone centre indicates that the $500 \mathrm{hPa}$ trough is slightly deeper in SST4 compared to in CNTL. Furthermore, the dipole of negative and positive anomalies downstream of the cyclone centre at $t=-48 \mathrm{~h}$ indicates that the $500 \mathrm{hPa}$ trough is sharper in SST4 compared to in CNTL. At $t=-24 \mathrm{~h}$ (Fig. 12b) an asymmetric dipole pattern is evident, which has small positive values upstream and larger negative values downstream of the cyclone centre. This indicates that the $500 \mathrm{hPa}$ trough is shifted downstream relative to the cyclone centre in SST4 compared to in CNTL but also that the trough is broader and extends more downstream in SST4 compared to CNTL. This pattern is also evident at $t=0$ and $t=+24 \mathrm{~h}$. The broader upper-level trough in SST4 is thus the likely reason why ascent due to vorticity 
(a) $t=-48 h$

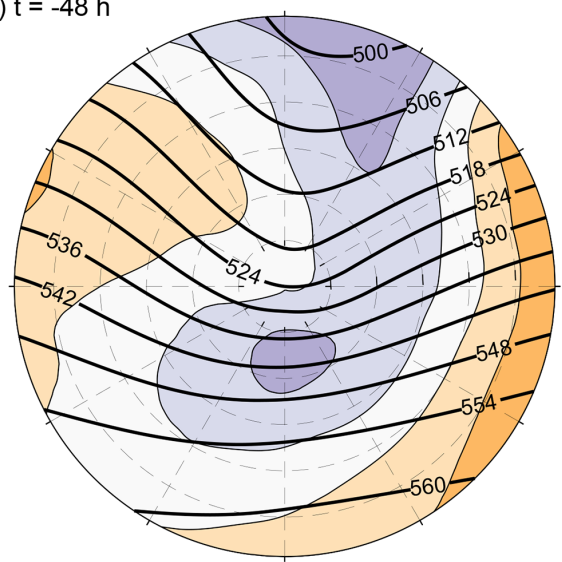

(c) $\mathrm{t}=\mathrm{Oh}$

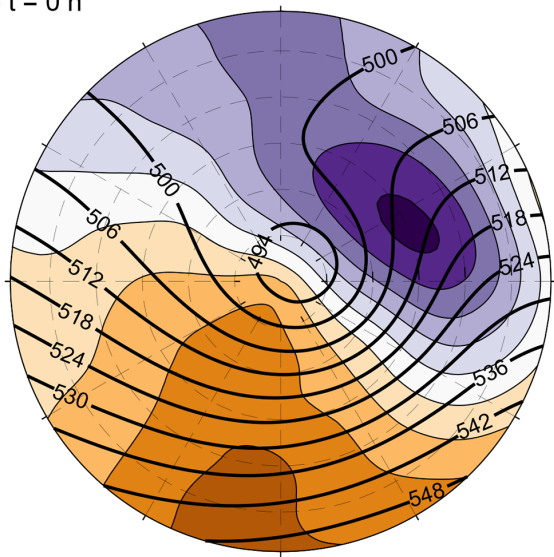

(b) $t=-24 h$

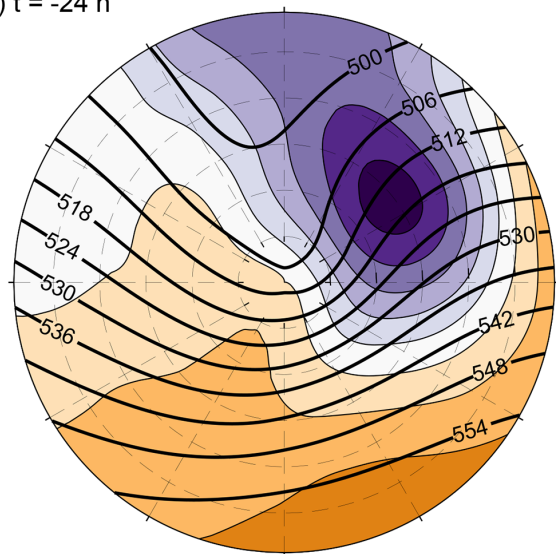

(d) $\mathrm{t}=+24 \mathrm{~h}$

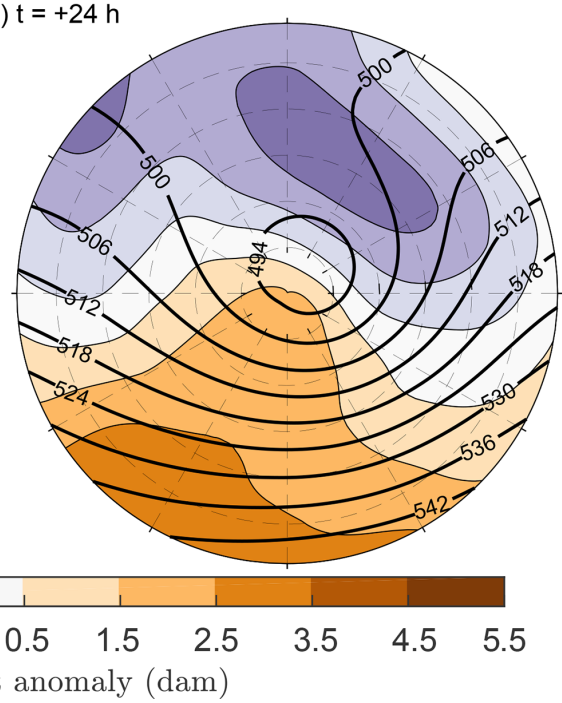

Figure 12. Composite mean of $500 \mathrm{hPa}$ geopotential height (dam) in CNTL (black contours) and the difference (SST4 - CNTL) in the cyclone relative anomalies at (a) $48 \mathrm{~h}$ before time of maximum intensity, (b) $24 \mathrm{~h}$ before time of maximum intensity, (c) time of maximum intensity, and (d) $24 \mathrm{~h}$ after the time of maximum intensity.

advection expands over a greater area downstream in SST4 compared to CNTL.

Ascent attributed to diabatic heating has a larger relative increase with warming in the warm front region compared to both dynamical terms (Fig. 11). At all offset times, ascent due to diabatic heating increases poleward of the maximum in the CNTL composite, which combined with the absence of any decrease in ascent results in an expansion of the area where diabatic heating contributes notably to ascent. Furthermore, in contrast to both thermal advection and vorticity advection, there are no coherent regions where descent due to diabatic heating has increased.

The spatial patterns of changes in ascent due to the different forcing mechanisms (Fig. 11) can be compared to the patterns of change in the total model output vertical velocity (Fig. 10). It can therefore be concluded that the increase in ascent poleward and downstream of the cyclone centre occurs due to a combination of all three processes. However, thermal advection and diabatic heating are responsible for most of the increase in ascent close to the cyclone centre, whereas vorticity advection is the main cause of the downstream expansion of the ascent field. The decrease in total ascent near the cyclone centre is found to be due to changes in spatial pattern of thermal advection and the position of the fronts. In the SST4 experiment, the warm front advances further ahead of the cyclone centre than in the CNTL, which results in weaker ascent due to warm air advection close to the cyclone centre. However, the decrease in ascent in the warm sector is a direct result of weaker ascent due to vorticity advection in this location, which arises as a consequence of the broader $500 \mathrm{hPa}$ trough. In contrast, the weaker descent immediately to the south of the cyclone centre is mainly due to weaker cold-air advection in SST4 compared to CNTL, which again relates to changing positions of the fronts. Finally, it should be noted that vertical velocities are likely to 
(a)

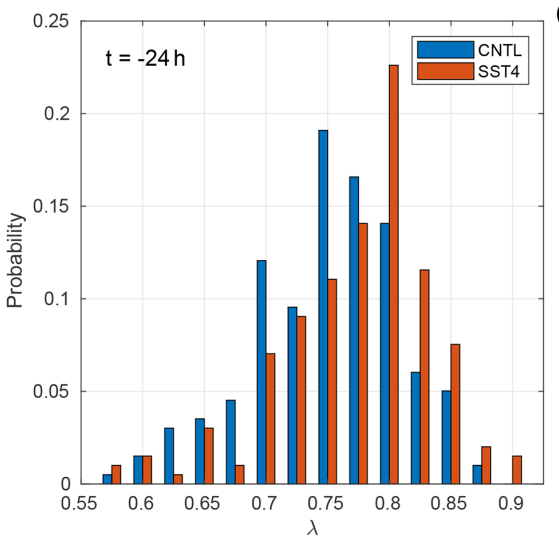

(b)

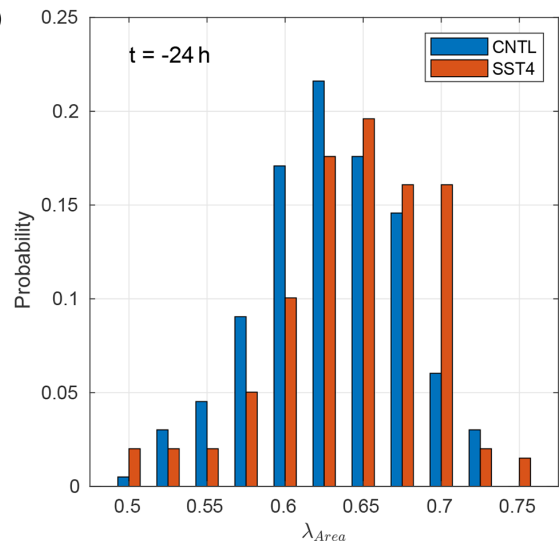

Figure 13. Normalized histograms of the (a) asymmetry parameter and (b) area asymmetry parameter (see text for more details) valid $24 \mathrm{~h}$ before the time of maximum intensity. Both histograms include values for the 200 strongest extra-tropical cyclones in both experiments. Blue shades show CNTL and red SST4.

be weaker for the same forcing in the warmer experiment due to an increase in tropospheric static stability (e.g. Fig. 3c).

\section{Asymmetry of vertical motion}

Ascent is stronger and occupies a smaller and narrower region than descent, both in the context of extra-tropical cyclones and globally. O'Gorman (2011) proposed two parameters, $\lambda$ and $\lambda_{\text {Area }}$, to quantify the asymmetry between upward and downward motions. The asymmetry parameter, $\lambda$, is given by

$\lambda \equiv \overline{\frac{\omega^{\prime} \omega^{\uparrow}}{\overline{\omega^{\prime 2}}}}$,

where in our study we define $\omega^{\prime}$ to be the perturbation from the mean vertical velocity $\left(\mathrm{Pa} \mathrm{s}^{-1}\right)$ calculated over the $12^{\circ}$ cyclone composite area. $\omega^{\uparrow}$ denotes taking the upward part of $\omega$ (i.e. applying a Heaviside function). The overbar denotes an average over the cyclone area. $\lambda_{\text {Area }}$ is an alternative (and more approximate) definition of asymmetry, which is based on geometric considerations. $\lambda_{\text {Area }}$ is given by

$\lambda_{\text {Area }}=\frac{1-a_{u}}{1-\bar{\omega} / \overline{\omega_{u}}}$,

where $a_{u}$ is the fraction of the total area (in this case the $12^{\circ}$ cyclone composite area) which is covered by ascent, $\overline{\omega_{u}}$ is the spatial average over the cyclone area of the ascent, and $\bar{\omega}$ is the spatial average of all vertical motions.

$\lambda$ and $\lambda_{\text {Area }}$ are both calculated for each of the 200 individual extra-tropical cyclones in both the CNTL and SST4 experiments at different offset times. Only vertical motions at one pressure level, $700 \mathrm{hPa}$, are considered. Figure 13 shows the distribution of both asymmetry parameters at $t=-24 \mathrm{~h}$, which reveals that there is considerable spread across the 200 strongest extra-tropical cyclones, particularly in $\lambda$, with values ranging from 0.55 to 0.9 in both experiments. However, it is also evident that there are larger values of both $\lambda$ and $\lambda_{\text {Area }}$ in SST4 compared to in CNTL. The mean values for both parameters are shown in Table 3. At $t=-24$, $t=0$, and $t=+24 \mathrm{~h}$, both $\lambda$ and $\lambda_{\text {Area }}$ are statistically significantly larger in SST4 compared to in CNTL; however the increases are small in magnitude. The fractional area of ascent $\left(a_{u}\right)$ is less than 0.5 at all offset times in both CNTL and SST4 (Table 3), indicating that regions of ascent are smaller relative to areas of descent. Warming does not cause large changes to the fractional area of ascent; a statistically significant decrease occurs at $t=0 \mathrm{~h}$, a statistically significant increase occurs at $t=+48 \mathrm{~h}$, and no significant changes occur at $t=-48,-24$, or $+24 \mathrm{~h}$. Thus the small changes in both asymmetry parameters occur due to changes in the ratio of mean descent to mean ascent. Small increases in the maximum ascent (minimum $\omega$, Table 2 ) are found and the mean vertical motion averaged over the $12^{\circ}$ cyclone area also becomes more negative in SST4 (Table 3), indicating either stronger ascent or weaker descent. The mean ascent, calculated over areas where $\omega<0$ in the $12^{\circ}$ cyclone area, strengthens slightly at $t=-24, t=0$, and $t=+24 \mathrm{~h}$ (Table 3) while the mean descent, averaged over areas where $\omega>0$ in the $12^{\circ}$ cyclone area, weakens slightly at all offset times. The magnitude of ratio between the mean upward velocity and mean downward velocity (i.e. $\left|\overline{\omega_{u}} / \overline{\omega_{\mathrm{d}}}\right|$ ) increases at all times, as does the ratio between the mean vertical motion and mean upward velocity (i.e. $\left|\bar{\omega} / \overline{\omega_{u}}\right|$ ), such that $\lambda$ increases with warming.

The magnitude of the mean increase in both $\lambda$ and $\lambda_{\text {Area }}$ is small; for example, at the time of maximum intensity $\lambda$ increases from 0.74 in CNTL to 0.77 in SST4, and $\lambda_{\text {Area }}$ increases from 0.64 in CNTL to 0.66 in SST4 (Table 3). This result differs slightly from the results of O'Gorman (2011), who, in idealized climate change simulations performed on an aqua planet, found that $\lambda$ has a value of $\sim 0.6$ and that 
Table 3. Mean values of $\lambda, \lambda_{\text {Area }}$ (see text for definitions), fractional area of ascent $\left(a_{u}\right)$, mean vertical motion $(\bar{\omega})$, mean ascent $\left(\overline{\omega_{u}}\right)$, and mean descent $\left(\overline{\omega_{\mathrm{d}}}\right)$ at $700 \mathrm{hPa}$ averaged over the strongest 200 extra-tropical cyclones in CNTL and in SST4 at different offset times.

\begin{tabular}{|c|c|c|c|c|c|c|c|c|c|c|c|c|}
\hline \multirow[b]{2}{*}{ Time (h) } & \multicolumn{6}{|c|}{ CNTL } & \multicolumn{6}{|c|}{ SST4 } \\
\hline & $\lambda_{\text {Area }}$ & $\lambda$ & $a_{u}$ & $\bar{\omega}$ & $\overline{\omega_{u}}$ & $\overline{\omega_{\mathrm{d}}}$ & $\lambda_{\text {Area }}$ & $\lambda$ & $a_{u}$ & $\bar{\omega}$ & $\overline{\omega_{u}}$ & $\overline{\omega_{\mathrm{d}}}$ \\
\hline-48 & 0.60 & 0.70 & 0.30 & 0.0011 & -0.1618 & 0.1083 & 0.59 & 0.70 & 0.30 & 0.0006 & -0.1578 & 0.1065 \\
\hline-24 & 0.63 & 0.75 & 0.41 & -0.0137 & -0.1922 & 0.1135 & 0.64 & 0.77 & 0.40 & -0.0178 & -0.1974 & 0.1091 \\
\hline 0 & 0.64 & 0.74 & 0.43 & -0.0183 & -0.1801 & 0.1021 & 0.66 & 0.77 & 0.42 & -0.0220 & -0.1890 & 0.0966 \\
\hline 24 & 0.60 & 0.69 & 0.45 & -0.0116 & -0.1322 & 0.0867 & 0.62 & 0.73 & 0.47 & -0.0162 & -0.1329 & 0.0805 \\
\hline 48 & 0.59 & 0.69 & 0.41 & -0.0066 & -0.1142 & 0.0777 & 0.60 & 0.71 & 0.42 & -0.0091 & -0.1053 & 0.0703 \\
\hline
\end{tabular}

it does not increase when the global mean surface temperature increases. Tamarin-Brodsky and Hadas (2019), however, find a small increase in $\lambda$ with warming and no changes to the fractional area of ascent $\left(a_{u}\right)$, which is in agreement with what we find here. Previously Booth et al. (2015) calculated $\lambda, \lambda_{\text {Area }}$, and $a_{u}$ in dry and moist baroclinic life cycle experiments and find that including moisture increases $\lambda$ from 0.58 to 0.74 , increases $\lambda_{\text {Area }}$ from 0.55 to 0.64 , and decreases $a_{u}$ from 0.45 to 0.40 . These changes are much more pronounced than those found in this study likely due to the relative difference in the two sets of experiments, e.g. a dry versus moist case compared to a moist case vs. a moist case with $4 \mathrm{~K}$ warming.

\section{Conclusions}

Aqua-planet simulations were performed with a state-of-theart, full-complexity atmospheric model (OpenIFS) to quantify how the number, characteristics, and structure of extratropical cyclones respond to horizontally uniform warming and to identify possible physical reasons for such changes. This simplified climate change experimental method was selected because it provides a very large sample size of cyclones for drawing statistically significant conclusions from and because the initial conditions and experimental design do not exert a strong control on the evolution of the model state.

The aqua-planet model set-up is capable of producing a zonal mean climate that is broadly similar to that observed on Earth. The response of the zonal mean temperature and zonal mean zonal wind to warming is in broad agreement with multi-model mean predictions from CMIP5 models. Namely, the greatest warming is observed in the tropical upper troposphere, the subtropical jet streams intensify, move upwards and polewards with warming, and the eddy-driven jet and mid-latitude storm track moves polewards with warming. The magnitude of the near-surface warming in the aquaplanet SST4 simulation compared to CNTL is approximately $4 \mathrm{~K}$, which is within the CMIP5 multi-model range predicted to occur by 2100 under the RCP 8.5 scenario.
Extra-tropical cyclones were tracked using an objective tracking algorithm which identifies localized maxima of $850 \mathrm{hPa}$ relative vorticity truncated to T42 spectral resolution. In both the control (CNTL) and warm (SST4) experiment about 3500 cyclone tracks were identified. Warming the SSTs did not change the cyclone lifetime and led to a $3.3 \%$ decrease in the total number of extra-tropical cyclones. Moreover, the median intensity of cyclones, as measured by the maximum $850 \mathrm{hPa}$ vorticity, does not change significantly when SSTs are warmed uniformly; however, the intensity distribution of extra-tropical cyclones broadens, resulting in more intense and more weaker cyclones. The median deepening rate of all extra-tropical cyclones did not change significantly with warming although the zonal mean Eady growth decreased by $5 \%-10 \%$ due to an increase in the hydrostatic stability. This apparent conflict arises as moisture acts to intensify the extra-tropical cyclones in these simulations whereas the Eady growth rate is a measure of dry baroclinicity. In addition, both extra-tropical cyclone genesis and lysis regions move poleward with warming, which is consistent with the poleward shift of the eddy-driven jet.

These results can be compared to those from previous idealized studies as well as to results obtained from fullcomplexity climate models. Our result that the maximum relative vorticity of the most extreme cyclones increases with warming is in agreement with results from previous aquaplanet simulations (Pfahl et al., 2015). When our results of extra-tropical cyclone intensity are compared to results based on coupled climate models, a complex picture emerges. Our result that the number of extreme cyclones increases with warming agrees with the results from Champion et al. (2011) and the Southern Hemisphere results of Chang et al. (2012) yet disagrees with the results from Bengtsson et al. (2009) and Catto et al. (2011), who both found that the number of intense storms in Europe and the North Atlantic is likely to decrease in the future.

Cyclone composites of the 200 strongest extra-tropical cyclones were created for both the CNTL and the SST4 experiments. The structure of both composite cyclones is qualitatively and even quantitatively very similar to composite cyclones created from reanalysis (Dacre et al., 2012) and historical climate model simulations (Catto et al., 2010). This 
strongly highlights the validity and usefulness of aqua-planet simulations. The aim of our composite analysis was to identify how the structure of the most intense extra-tropical cyclones responds to warming. The main focus was on how precipitation and vertical motion respond to warming, and the omega equation was utilized to assess changes to vertical motion forced by thermal and vorticity advection and attributable to diabatic heating. The main results of how the structure of the 200 most intense extra-tropical cyclones change with warming include the following.

1. The total column water vapour (TCWV) increases everywhere within a $12^{\circ}$ radius of the cyclone centre, and the meridional TCWV gradient increases. The largest absolute increases in TCWV occur in the warm sector, whereas the largest relative increases occur poleward of the warm front.

2. The $900-700 \mathrm{hPa}$ layer average potential vorticity increases at all stages of the cyclone evolution everywhere within a $12^{\circ}$ radius of the cyclone centre. The small absolute increases away from the cyclone centre result from increasing stratification.

3. The $900 \mathrm{hPa}$ wind speed increases in particular in the warm sector and thus the size of the area exposed to wind speeds above a certain fixed threshold increases.

4. The low-level potential vorticity, total and large-scale precipitation, and ascent at $700 \mathrm{hPa}$ ahead of the warm front increase at all times of the cyclone life cycle, which occurs due to an increase in ascent forced by thermal advection and an increase in how diabatic processes enhance ascent.

5. The area of ascent downstream of the cyclone centre expands due to increased ascent forced by vorticity advection. This is related to a downstream shift and broadening of the $500 \mathrm{hPa}$ trough.

6. The maximum values of ascent at $700 \mathrm{hPa}$ decrease slightly due to vorticity and thermal advection during the cyclone intensification phase, and the maximum value of ascent increases slightly due to diabatic heating.

7. The asymmetry of vertical motion $\left(\lambda\right.$ and $\left.\lambda_{\text {Area }}\right)$ increases slightly with warming, and the fractional area of ascent does not notably change. The small increases in asymmetry arise as the absolute magnitude of the increase in ascent is greater than the absolute decrease in the magnitude of the descent.

First, these results show that ascent becomes slightly more diabatically driven in the warmer experiment compared to the CNTL experiment and that cyclone-related precipitation increases by up to $50 \%$ - a value much larger than predicted for global precipitation amounts. Second, these results indicate that the spatial structure of the most intense extra-tropical cyclones does change with warming. The localized maxima of low-level PV, $900 \mathrm{hPa}$ wind speed, maximum precipitation, and vertical velocity associated with the warm front all move northeast relative to the centre of the cyclone. This demonstrates that in the warmer experiment the warm front is farther poleward and downstream of the cyclone centre than in CNTL. Furthermore, the area of ascent also increased with warming, particularly in the downstream region due to changes in ascent forced by vorticity advection and ultimately a broader trough at $500 \mathrm{hPa}$ in SST4 compared to in the CNTL experiment.

The cyclone composite analysis revealed that precipitation increased everywhere relative to the cyclone centre with warming. The same result was obtained by Yettella and Kay (2017), who analysed a 30-member initial condition climate model ensemble. Furthermore, Yettella and Kay (2017) find that precipitation in a cyclone composite of Northern Hemisphere wintertime extra-tropical cyclones increases from maximum values of $\sim 9 \mathrm{~mm}$ per day to $11 \mathrm{~mm} \mathrm{~d}^{-1}$ in the far-future (2081-2100) simulations forced with RCP8.5. The relative increase is thus smaller than that found in our aqua-planet simulations, which is likely due to the fact that in the real world evaporation over land is limited, whereas there is always a limitless source of moisture at the surface in an aqua planet.

The most striking similarity found between our results and previous studies is the downstream shift in the low-level vorticity anomaly and precipitation relative to the cyclone centre. Kirshbaum et al. (2018) and Tierney et al. (2018) both find very similar results of how extra-tropical cyclone structure responds to warming in their baroclinic life cycle experiments. Kirshbaum et al. (2018) show that with increasing environmental temperature the cyclonic potential vorticity associated with the warm front strengthened and moved downstream, while Tierney et al. (2018) show that in warmer simulations the upper-level PV anomaly is much farther west relative to the low-level PV anomaly than in colder and drier simulations. Thus, this spatial change appears to be a robust feature of how extra-tropical cyclones respond to warming. We thus speculate that in a warmer climate the classical coupling and mutual intensification of lower- and upper-level anomalies may be disrupted and that extra-tropical cyclone dynamics and associated weather may be notably different.

This study prioritized in-depth understanding of changes to the dynamics and structure of extra-tropical cyclones with warming, rather than quantifying extra-tropical cyclone structure in specific future climate scenarios. Thus, the simulations included numerous simplifications, and consequently there are some caveats to this study. First, the aqua-planet simulations contain no polar amplification, and thus the lowlevel temperature gradient does not change with warming. Chang et al. (2012) show that CMIP5 models predict a sig- 
nificant increase in the frequency of extreme cyclones during the winter in the Southern Hemisphere, a result which is in general agreement with our aqua-planet results. This means that (1) our results may be more applicable to the Southern Hemisphere and (2) our results, together with further additional simulations, could be used to ascertain the impact of polar amplification on extra-tropical cyclone intensity. Second, as there is no land in our simulations, the potential impact of differential changes in land-sea temperatures on extra-tropical cyclone dynamics is not considered, meaning that our results are likely more applicable to oceanic extratropical cyclones. Third, we applied uniform SST warming, which neglects localized oceanic cooling that occurs in the northern North Atlantic and parts of the high-latitude Southern Ocean in several CMIP5 models (Fig. 12.9, Collins et al., 2013). Fourth, the simulations were performed at a resolution more typical of a climate model $(125 \mathrm{~km})$ than of a numerical weather prediction model, meaning that in both the CNTL and SST4 experiments extra-tropical cyclones may be weaker and precipitation areas broader than if the simulations had been conducted at higher resolution. Nevertheless it appears unlikely that repeating the experiments at higher resolution would fundamentally change the main conclusions as Jung et al. (2012) find that increasing the resolution of the IFS from T159 to T1279 only increases the amount of precipitation by $6 \%$ and does not alter the ratio of convective to large-scale precipitation. Lastly, cyclone composites were created from the strongest 200 storms and clearly the results would differ if we considered a larger or smaller number.

We conclude by noting that the results obtained here can be used as a stepping stone to better understand predictions from coupled climate models of how the structure of extratropical cyclones is likely to change in the future. A logical next step would be to analyse climate model projections for evidence of the downstream shift relative to the cyclone centre of increased low-level potential vorticity, vertical velocity, and precipitation.

Code availability. OpenIFS is available under license from the European Centre for Medium-Range Weather Forecasts (ECMWF). See https://confluence.ecmwf.int/display/OIFS (ECMWF, 2015) for more details. Information on how to obtain the cyclone identification and tracking algorithm (TRACK) can be found from http:// www.nerc-essc.ac.uk/ kih/TRACK/Track.html. The version of the omega equation code applied here is available from Mika Rantanen on request.

Supplement. The supplement related to this article is available online at: https://doi.org/10.5194/wcd-1-1-2020-supplement.

Author contributions. VAS designed and performed the numerical experiments, analysed the data, and wrote the paper. MR performed the omega equation calculations. PH performed the cyclone track- ing and ran the cyclone composite code. JR and HJ provided guidance on interpreting the results. All authors commented on the paper.

Competing interests. The authors declare that they have no conflict of interest.

Acknowledgements. We acknowledge ECMWF for making the OpenIFS model available and CSC - IT Center for Science Ltd. for the allocation of computational resources. We thank Glenn Carver, Filip Ván^a, and Gabriela Szépszó for assistance with OpenIFS and for creating the initial conditions for the simulations. We also thank Kevin Hodges for providing the cyclone tracking code, TRACK, and Helen Dacre for providing the cyclone composite code.

Financial support. This research has been supported by the Academy of Finland (grant no. 307331).

Review statement. This paper was edited by Sebastian Schemm and reviewed by two anonymous referees.

\section{References}

Bengtsson, L., Hodges, K. I., and Keenlyside, N.: Will extratropical storms intensify in a warmer climate?, J. Climate, 22, 22762301, 2009.

Binder, H., Boettcher, M., Joos, H., and Wernli, H.: The role of warm conveyor belts for the intensification of extratropical cyclones in Northern Hemisphere winter, J. Atmos. Sci., 73, 39974020, 2016.

Bjerknes, J.: On the structure of moving cyclones, Mon. Weather Rev., 47, 95-99, 1919.

Booth, J. F., Wang, S., and Polvani, L.: Midlatitude storms in a moister world: Lessons from idealized baroclinic life cycle experiments, Clim. Dynam., 41, 787-802, 2013.

Booth, J. F., Polvani, L., O'Gorman, P. A., and Wang, S.: Effective stability in a moist baroclinic wave, Atmos. Sci. Lett., 16, 56-62, 2015.

Boutle, I. A., Belcher, S. E., and Plant, R. S.: Moisture transport in midlatitude cyclones, Q. J. Roy. Meteorol. Soc., 137, 360-373, 2011.

Browning, K. A., Hardman, M. E., Harrold, T. W., and Pardoe, C. W.: The structure of rainbands within a mid-latitude depression, Q. J. Roy. Meteorol. Soc., 99, 215-231, 1973.

Carlson, T. N.: Airflow through midlatitude cyclones and the comma cloud pattern, Mon. Weather Rev., 108, 1498-1509, 1980.

Catto, J. L., Shaffrey, L. C., and Hodges, K. I.: Can climate models capture the structure of extratropical cyclones?, J. Climate, 23, 1621-1635, 2010.

Catto, J. L., Shaffrey, L. C., and Hodges, K. I.: Northern Hemisphere extratropical cyclones in a warming climate in the 
HiGEM high-resolution climate model, J. Climate, 24, 53365352, 2011.

Champion, A. J., Hodges, K. I., Bengtsson, L. O., Keenlyside, N. S., and Esch, M.: Impact of increasing resolution and a warmer climate on extreme weather from Northern Hemisphere extratropical cyclones, Tellus A, 63, 893-906, 2011.

Chang, E. K., Guo, Y., and Xia, X.: CMIP5 multimodel ensemble projection of storm track change under global warming, J. Geophys. Res., 117, D23118, https://doi.org/10.1029/2012JD018578, 2012.

Collins, M., Knutti, R., Arblaster, J., Dufresne, J.-L., Fichefet, T., Friedlingstein, P., Gao, X., Gutowski, W. J., Johns, T., Krinner, G., Shongwe, M., Tebaldi, C., Weaver, A. J., and Wehner, M.: Long-term Climate Change: Projections, Commitments and Irreversibility, in: Climate Change 2013: The Physical Science Basis, Contribution of Working Group I to the Fifth Assessment Report of the Intergovernmental Panel on Climate Change, chap. 12, edited by: Stocker, T. F., Qin, D., Plattner, G.-K., Tignor, M., Allen, S. K., Boschung, J., Nauels, A., Xia, Y., Bex, V., and Midgley, P. M., Cambridge University Press, Cambridge, UK and New York, NY, USA, 1029-1136, https://doi.org/10.1017/CBO9781107415324.024, 2013.

Dacre, H. F., Hawcroft, M. K., Stringer, M. A., and Hodges, K. I.: An extratropical cyclone atlas: A tool for illustrating cyclone structure and evolution characteristics, B. Am. Meteorol. Soc., 93, 1497-1502, 2012.

Eckhardt, S., Stohl, A., Wernli, H., James, P., Forster, C., and Spichtinger, N.: A 15-year climatology of warm conveyor belts, J. Climate, 17, 218-237, 2004.

ECMWF: Part IV: Physical Processes, in: chap. 4, IFS Documentation, operational implementation 12 May 2015, ECMWF, Reading, UK, 1-190, 2015.

Field, P. R. and Wood, R.: Precipitation and cloud structure in midlatitude cyclones, J. Climate, 20, 233-254, 2007.

Fink, A. H., Brücher, T., Ermert, V., Krüger, A., and Pinto, J. G.: The European storm Kyrill in January 2007: synoptic evolution, meteorological impacts and some considerations with respect to climate change, Nat. Hazards Earth Syst. Sci., 9, 405-423, https://doi.org/10.5194/nhess-9-405-2009, 2009.

Govekar, P. D., Jakob, C., and Catto, J.: The relationship between clouds and dynamics in Southern Hemisphere extratropical cyclones in the real world and a climate model, J. Geophys. Res., 119, 6609-6628, 2014.

Harrold, T. W.: Mechanisms influencing the distribution of precipitation within baroclinic disturbances, Q. J. Roy. Meteorol. Soc., 99, 232-251, 1973.

Hawcroft, M. K., Shaffrey, L. C., Hodges, K. I., and Dacre, H. F.: How much Northern Hemisphere precipitation is associated with extratropical cyclones?, Geophys. Res. Lett., 39, L24809, https://doi.org/10.1029/2012GL053866, 2012.

Hodges, K. I.: A general method for tracking analysis and its application to meteorological data, Mon. Weather Rev., 122, 25732586, 1994.

Hodges, K. I.: Feature tracking on the unit sphere, Mon. Weather Rev., 123, 3458-3465, 1995.

Holton, J. R. and Hakim, G. J.: An introduction to dynamic meteorology, in: vol. 88, Academic Press, Oxford, UK, 2012.
Hoskins, B. J. and Hodges, K. I.: New perspectives on the Northern Hemisphere winter storm tracks, J. Atmos. Sci., 59, 1041-1061, 2002.

Hoskins, B. J., McIntyre, M., and Robertson, A. W.: On the use and significance of isentropic potential vorticity maps, Q. J. Roy. Meteorol. Soc., 111, 877-946, 1985.

Jung, T., Miller, M. J., Palmer, T. N., Towers, P., Wedi, N., Achuthavarier, D., Adams, J. M., Altshuler, E. L., Cash, B. A., Kinter III, J. L., Marx, L., Stan, C., and Hodges, K. I.: Highresolution global climate simulations with the ECMWF model in Project Athena: Experimental design, model climate, and seasonal forecast skill, J. Climate, 25, 3155-3172, 2012.

Kirshbaum, D., Merlis, T., Gyakum, J., and McTaggart-Cowan, R.: Sensitivity of idealized moist baroclinic waves to environmental temperature and moisture content, J. Atmos. Sci., 75, 337-360, 2018.

Lau, W. K.-M., Wu, H.-T., and Kim, K.-M.: A canonical response of precipitation characteristics to global warming from CMIP5 models, Geophys. Res. Lett., 40, 3163-3169, 2013.

Michaelis, A. C., Willison, J., Lackmann, G. M., and Robinson, W. A.: Changes in Winter North Atlantic Extratropical Cyclones in High-Resolution Regional Pseudo - Global Warming Simulations, J. Climate, 30, 6905-6925, 2017.

Naud, C. M., Del Genio, A. D., Bauer, M., and Kovari, W.: Cloud vertical distribution across warm and cold fronts in CloudSatCALIPSO data and a general circulation model, J. Climate, 23, 3397-3415, 2010.

Naud, C. M., Booth, J. F., Lebsock, M., and Grecu, M.: Observational constraint for precipitation in extratropical cyclones: Sensitivity to data sources, J. Appl. Meteorol. Clim., 57, 991-1009, 2018.

Neale, R. B. and Hoskins, B. J.: A standard test for AGCMs including their physical parametrizations: I: The proposal, Atmos. Sci. Lett., 1, 101-107, 2000.

Neu, U., Akperov, M. G., Bellenbaum, N., Benestad, R., Blender, R., Caballero, R., Cocozza, A., Dacre, H. F., Feng, Y., Fraedrich, K., Grieger, J., Gulev, S., Hanley, J., Hewson, T., Inatsu, M., Keay, K., Kew, S. F., Kindem, I., Leckebusch, G. C., Liberato, M. L. R., Lionell, P., Mokhov, I. I., Pinto, J. G., Raible, C. C., Reale, M., Rudevaia, I., Schuster, M., Simmonds, I., Sinclair, M., Sprenger, M., Tilinina, N. D., Trigo, I. F., Ulbrich, S., Ulbrich, U., Wang, X. L., and Wernli, H.: IMILAST: A community effort to intercompare extratropical cyclone detection and tracking algorithms, B. Am. Meteorol. Soc., 94, 529-547, 2013.

O'Gorman, P. A.: The effective static stability experienced by eddies in a moist atmosphere, J. Atmos. Sci., 68, 75-90, 2011.

Pfahl, S., O'Gorman, P. A., and Singh, M. S.: Extratropical cyclones in idealized simulations of changed climates, J. Climate, 28, 9373-9392, 2015.

Räisänen, J.: Factors affecting synoptic-scale vertical motions: A statistical study using a generalized omega equation, Mon. Weather Rev., 123, 2447-2460, 1995.

Rantanen, M., Räisänen, J., Lento, J., Stepanyuk, O., Räty, O., Sinclair, V. A., and Järvinen, H.: OZO v.1.0: software for solving a generalised omega equation and the Zwack-Okossi height tendency equation using WRF model output, Geosci. Model Dev., 10, 827-841, https://doi.org/10.5194/gmd-10-827-2017, 2017.

Rantanen, M., Räisänen, J., Sinclair, V. A., and Järvinen, H.: Sensitivity of idealised baroclinic waves to mean atmospheric temper- 
ature and meridional temperature gradient changes, Clim. Dynam., 52, 2703-2719, 2019.

Schemm, S. and Wernli, H.: The linkage between the warm and the cold conveyor belts in an idealized extratropical cyclone, J. Atmos. Sci., 71, 1443-1459, 2014.

Schemm, S., Wernli, H., and Papritz, L.: Warm conveyor belts in idealized moist baroclinic wave simulations, J. Atmos. Sci., 70, 627-652, 2013.

Schultz, D. M., Bosart, L. F., Colle, B. A., Davies, H. C., Dearden, C., Keyser, D., Martius, O., Roebber, P. J., Steenburgh, W. J., Volkert, H., and Winters, A. C.: Extratropical Cyclones: A Century of Research on Meteorology's Centerpiece, Meteorol. Monogr., 59, 16.1-16.56, 2019.

Shapiro, M. A. and Keyser, D.: Fronts, jet streams and the tropopause., in: Extratropical Cyclones, The Erik Palmén Memorial Volume, Amer. Meteor. Soc., Boston, USA, 167-191, 1990.

Shepherd, T. G.: Atmospheric circulation as a source of uncertainty in climate change projections, Nat. Geosci., 7, 703-708, https://doi.org/10.1038/ngeo2253, 2014.

Simmonds, I. and Keay, K.: Mean Southern Hemisphere extratropical cyclone behavior in the 40-year NCEP-NCAR reanalysis, J. Climate, 13, 873-885, 2000.

Simmons, A. J. and Hoskins, B. J.: The life cycles of some nonlinear baroclinic waves, J. Atmos. Sci., 35, 414-432, 1978.

Sinclair, V. A. and Keyser, D.: Force balances and dynamical regimes of numerically simulated cold fronts within the boundary layer, Q. J. Roy. Meteorol. Soc., 141, 2148-2164, 2015.

Stoelinga, M. T.: A potential vorticity-based study of the role of diabatic heating and friction in a numerically simulated baroclinic cyclone, Mon. Weather Rev., 124, 849-874, 1996.
Tamarin-Brodsky, T. and Hadas, O.: The asymmetry of vertical velocity in current and future climate, Geophys. Res. Lett., 46, 374382, 2019.

Thorncroft, C., Hoskins, B., and McIntyre, M.: Two paradigms of baroclinic-wave life-cycle behaviour, Q. J. Roy. Meteorol. Soc., 119, 17-55, 1993.

Tierney, G., Posselt, D. J., and Booth, J. F.: An examination of extratropical cyclone response to changes in baroclinicity and temperature in an idealized environment, Clim. Dynam., 51, 3829, https://doi.org/10.1007/s00382-018-4115-5, 2018.

Wernli, H. and Davies, H. C.: A Lagrangian-based analysis of extratropical cyclones. I: The method and some applications, Q. J. Roy. Meteorol. Soc., 123, 467-489, 1997.

Wernli, H. and Schwierz, C.: Surface cyclones in the ERA-40 dataset (1958-2001). Part I: Novel identification method and global climatology, J. Atmos. Sci., 63, 2486-2507, 2006.

Yettella, V. and Kay, J. E.: How will precipitation change in extratropical cyclones as the planet warms? Insights from a large initial condition climate model ensemble, Clim. Dynam., 49, 17651781, 2017.

Zappa, G., Shaffrey, L. C., and Hodges, K. I.: The ability of CMIP5 models to simulate North Atlantic extratropical cyclones, J. Climate, 26, 5379-5396, 2013a.

Zappa, G., Shaffrey, L. C., Hodges, K. I., Sansom, P. G., and Stephenson, D. B.: A multimodel assessment of future projections of North Atlantic and European extratropical cyclones in the CMIP5 climate models, J. Climate, 26, 5846-5862, $2013 \mathrm{~b}$. 